\title{
Baseline for ostracod-based northwestern Pacific and Indo-Pacific shallow-marine paleoenvironmental reconstructions: ecological modeling of species distributions
}

\author{
Yuanyuan Hong $^{1,2}$, Moriaki Yasuhara ${ }^{1,2}$, Hokuto Iwatani ${ }^{1,2}$, and Briony Mamo ${ }^{1,2}$ \\ ${ }^{1}$ School of Biological Sciences, The University of Hong Kong, Pok Fu Lam Road, Hong Kong SAR, China \\ ${ }^{2}$ Swire Institute of Marine Science, The University of Hong Kong, Cape d'Aguilar Road, Shek O, Hong Kong SAR, China
}

Correspondence: Yuanyuan Hong (oocirclr@gmail.com) and Moriaki Yasuhara (moriakiyasuhara@gmail.com)

Received: 11 September 2018 - Discussion started: 15 October 2018

Revised: 28 December 2018 - Accepted: 4 January 2019 - Published: 1 February 2019

\begin{abstract}
Fossil ostracods have been widely used for Quaternary paleoenvironmental reconstructions, especially in marginal marine environments (e.g., for water depth, temperature, salinity, oxygen levels, pollution). But our knowledge of indicator species autoecology, the base of paleoenvironmental reconstructions, remains limited and commonly lacks robust statistical support and comprehensive comparison with environmental data. We analyzed marginal marine ostracod taxa at 52 sites in Hong Kong for which comprehensive environmental data are available. We applied linear regression models to reveal relationships between species distribution and environmental factors for 18 common taxa (mainly species, a few genera) in our Hong Kong dataset and identified indicator species of environmental parameters. For example, Sinocytheridea impressa, a widely distributed euryhaline species throughout the East and South China Sea and the Indo-Pacific, indicates eutrophication and bottom-water hypoxia. Neomonoceratina delicata, a widely known species from nearshore and estuarine environments in the East and South China Sea and the Indo-Pacific, indicates heavy metal pollution and increased turbidity. The 18 taxa used for this study are widely distributed geographically and divided into the following groups: widespread (throughout the northwestern Pacific and Indo-Pacific regions), temperate (South China Sea to Russia (Sea of Japan coast) and Japan), subtropical (Indo-Pacific to the East China Sea), tropical (Indo-Pacific and South China Sea), and globally distributed. With statistical support from ecological modeling and comprehensive environmental data, these results provide a robust baseline for ostracod-based Quaternary-Anthropocene paleoenvironmental reconstruc-
\end{abstract}

tions in the tropical-extratropical northwestern Pacific and Indo-Pacific.

\section{Highlights.}

1. We provide a robust baseline for ostracod-based (microscopic Arthropods) paleoenvironmental reconstructions from Quaternary and Anthropocene marginal marine sediments.

2. The studied species have wide distributions over the tropics and extratropics of the northwestern Pacific and Indo-Pacific.

3. Ecological modeling has established ostracod species as reliable indicators for paleoenvironmental reconstructions.

\section{Introduction}

Because of their small size, high abundance, and excellent fossil record, fossil ostracods (microcrustaceans) have been widely used to reconstruct Quaternary environmental conditions including water depth, salinity, temperature, oxygen, and pollution, especially in marginal marine sediments (Boomer and Eisenhauer, 2002; Cronin, 2015; Frenzel and Boomer, 2005; Horne et al., 2012; Ruiz et al., 2005). In the northwestern Pacific and Indo-Pacific there are numerous deltas (Woodroffe et al., 2006) hosting accumulated Holocene marine sediments. Many studies have reconstructed the depositional environments of these sediments 


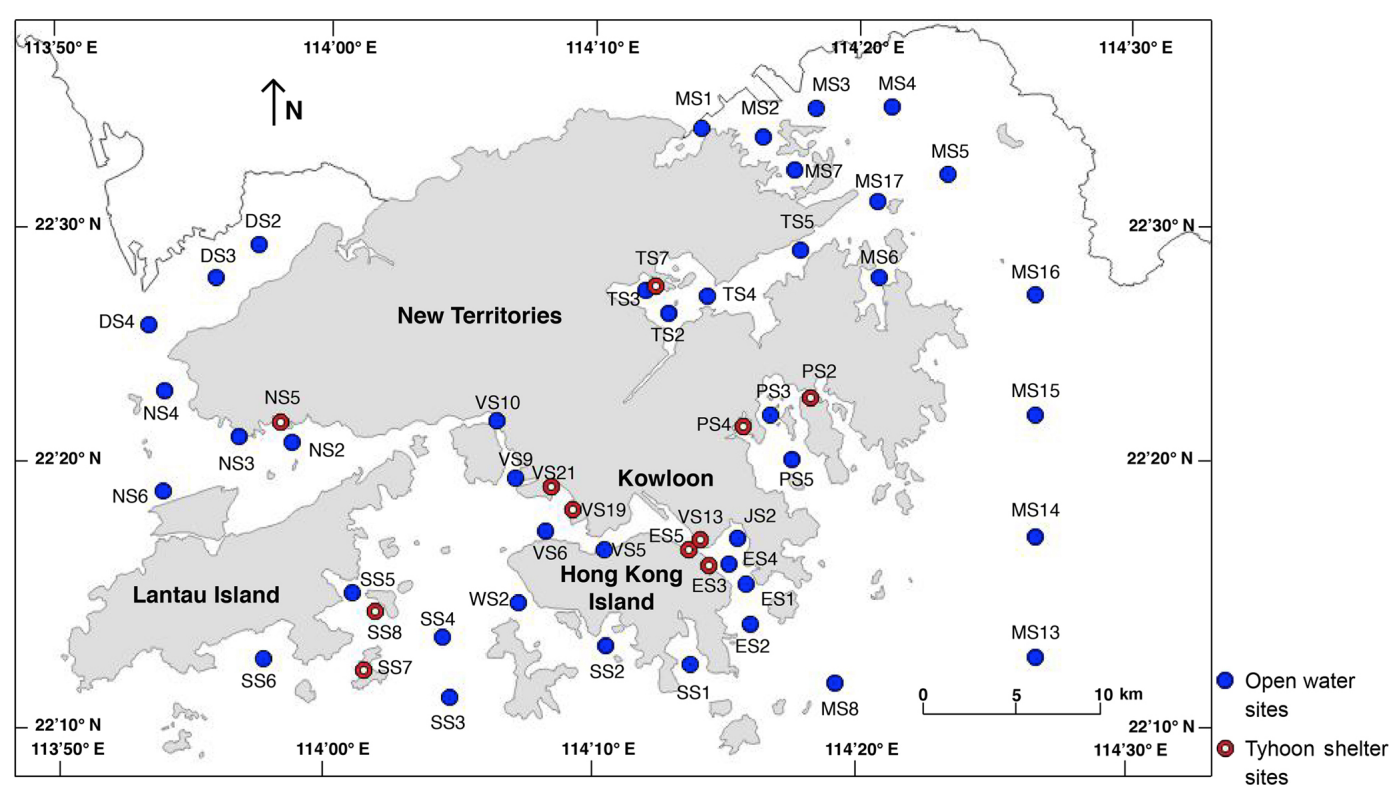

Figure 1. Locality map showing the 52 sampling sites across Hong Kong, including 41 open water sites (blue dots) and 11 typhoon shelter sites (red open dots). From west to east, DS: Deep Bay; NS: northwestern waters; SS: southern waters; VS: Victoria Harbour; ES: eastern buffer; JS: Junk Bay; TS: Tolo Harbour; PS: Port Shelter; MS: Mirs Bay.

(Alberti et al., 2013; Dong et al., 2012; Irizuki et al., 2015b; Tanaka et al., 2011; Yasuhara and Seto, 2006; Yasuhara et al., 2005; Zhou et al., 2015; Wang et al., 2018). Due to high sedimentation rates $\left(>1 \mathrm{~cm}\right.$ year $^{-1}$ ), fossil ostracods from these sediments allow for the high-resolution reconstruction of human-induced environmental changes (pollution, eutrophication, bottom oxygen depletion) over the past century (Irizuki et al., 2011, 2015a, 2018; Yasuhara et al., 2003, 2007).

Many have evaluated the autoecology of ostracod indicator species as the basis for paleoenvironmental reconstructions (Hazel, 1988; Irizuki et al., 2003; Ozawa et al., 2004; Stepanova et al., 2003; Wang et al., 1988; Yasuhara and Seto, 2006; Zhao, 1984; Zhao and Wang, 1988a, b). Yet these studies tend to focus on only one or a few targeted environmental factor(s) and lack rigorous statistical evaluation, particularly statistical modeling, a common approach in contemporary ecology. This is probably due to the fact that comprehensive environmental datasets are often unavailable and an ecological modeling approach (especially regression modeling and model selection) has not been common in this field of micropaleontology.

Hong Kong constitutes an ideal location for a marine ecological modeling approach in the northwestern Pacific and Indo-Pacific regions because of an extensive and intensive marine environmental monitoring program and a subtropical location where tropical and temperate species coexist. This program provides robust datasets for ecological modeling, and the subtropical location allows for the investigation of species with different latitudinal and geographi- cal distributions. We employed regression modeling of Hong Kong shallow-marine ostracod species to show statistical relationships between species abundance, distribution, and environmental factors. This study allows for the autoecology and statistical evaluation of common tropical and extratropical species, providing a baseline for ostracod-based shallowmarine paleoenvironmental reconstructions of the northwestern Pacific and Indo-Pacific regions.

\section{Study area}

Hong Kong is situated at the southeastern corner of the Pearl River (Zhujiang) Delta and has an area of $2500 \mathrm{~km}^{2}$ (Fig. 1) at $22^{\circ} 12.021^{\prime}$ to $22^{\circ} 33.817^{\prime} \mathrm{N}$ latitude, $113^{\circ} 53.388^{\prime}$ to $114^{\circ} 26.920^{\prime} \mathrm{E}$ longitude. It is an ideal location to study natural and/or anthropogenic impacts on benthic communities due to its complex hydrology and long history of human influence. Western Hong Kong is affected by input from the Pearl River, particularly during the summer heavy rainfall (Morton and $\mathrm{Wu}, 1975$ ) when surface water salinity is strongly influenced by rainfall. Eastern Hong Kong waters are mainly marine and derived from the South China Sea. As one of the most urbanized coastal areas in the world, human activities including rapid industrialization, sewage discharge, trawling, dredging, and land reclamation have led to a deteriorated marine benthic ecosystem (Blackmore, 1998; Hodgkiss and Yim, 1995; Hong et al., 2017; Hu et al., 2008; Morton, 1996; Morton and Blackmore, 2001; Owen and Sandhu, 2000; Shin, 2011; Tanner et al., 2000). 

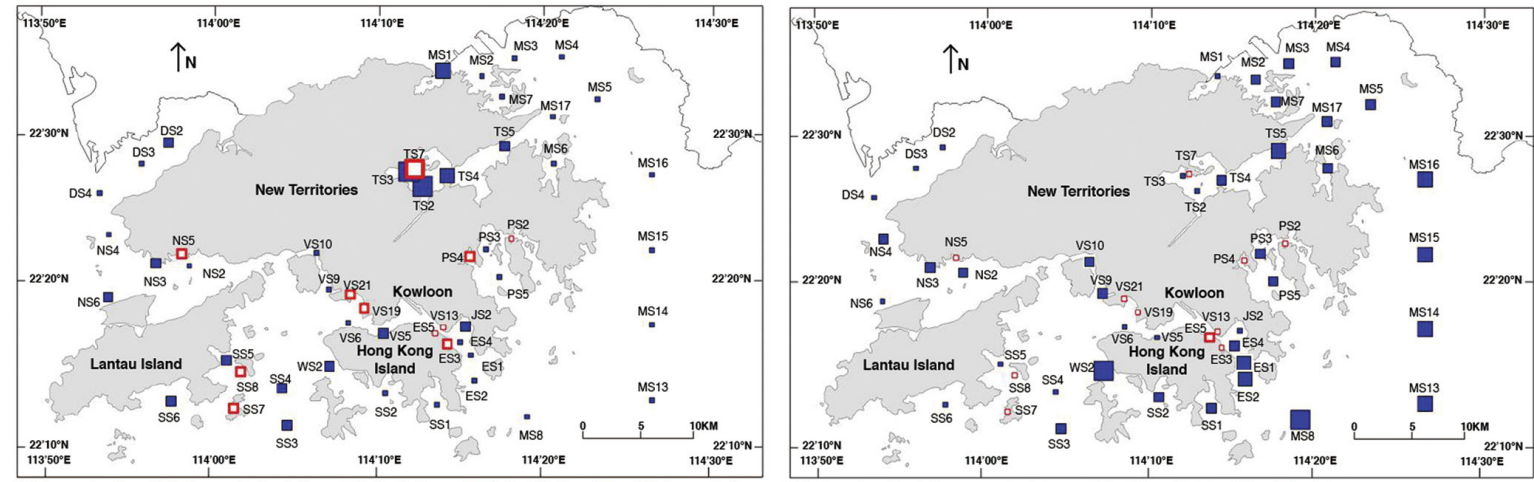

Chlorophyll $a$ (Chl) $\quad: 1.0-4.0 \quad \nabla 4.1-8.0 \quad \square 8.1-12.0 \quad \square>12.0 \mu \mathrm{g} \mathrm{L}^{-1}$
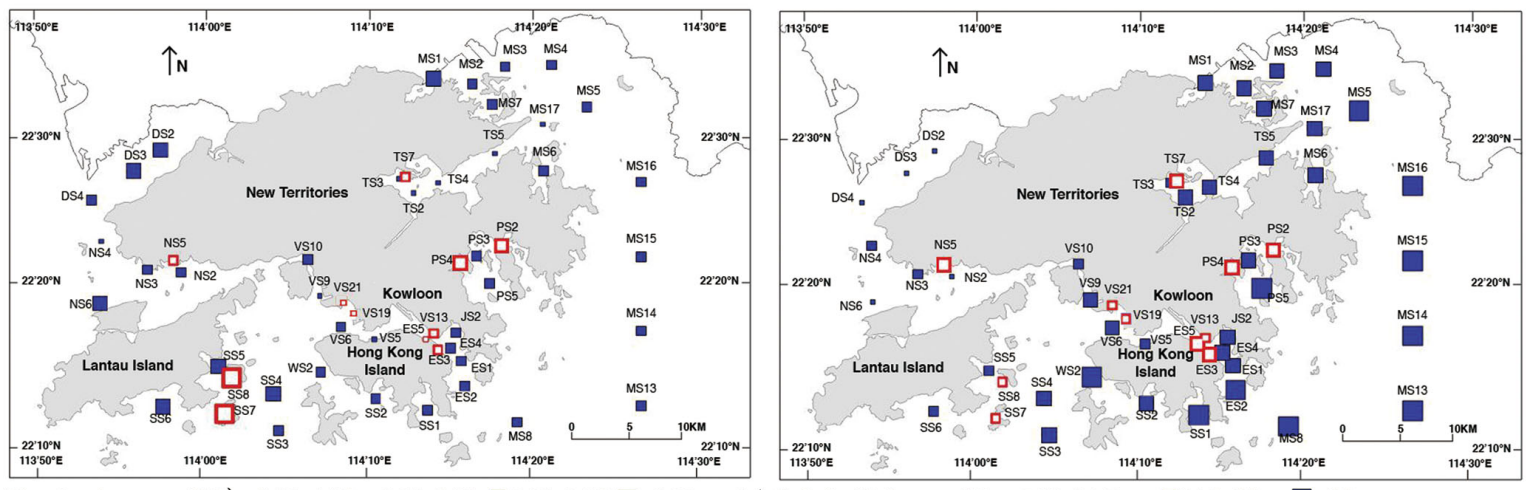

Dissolved oxygen (DO) $\cdot 2.95-4.00=4.01-5.00 \square 5.01-6.00 \square>6.00 \mathrm{mg} \mathrm{L}^{-1}$
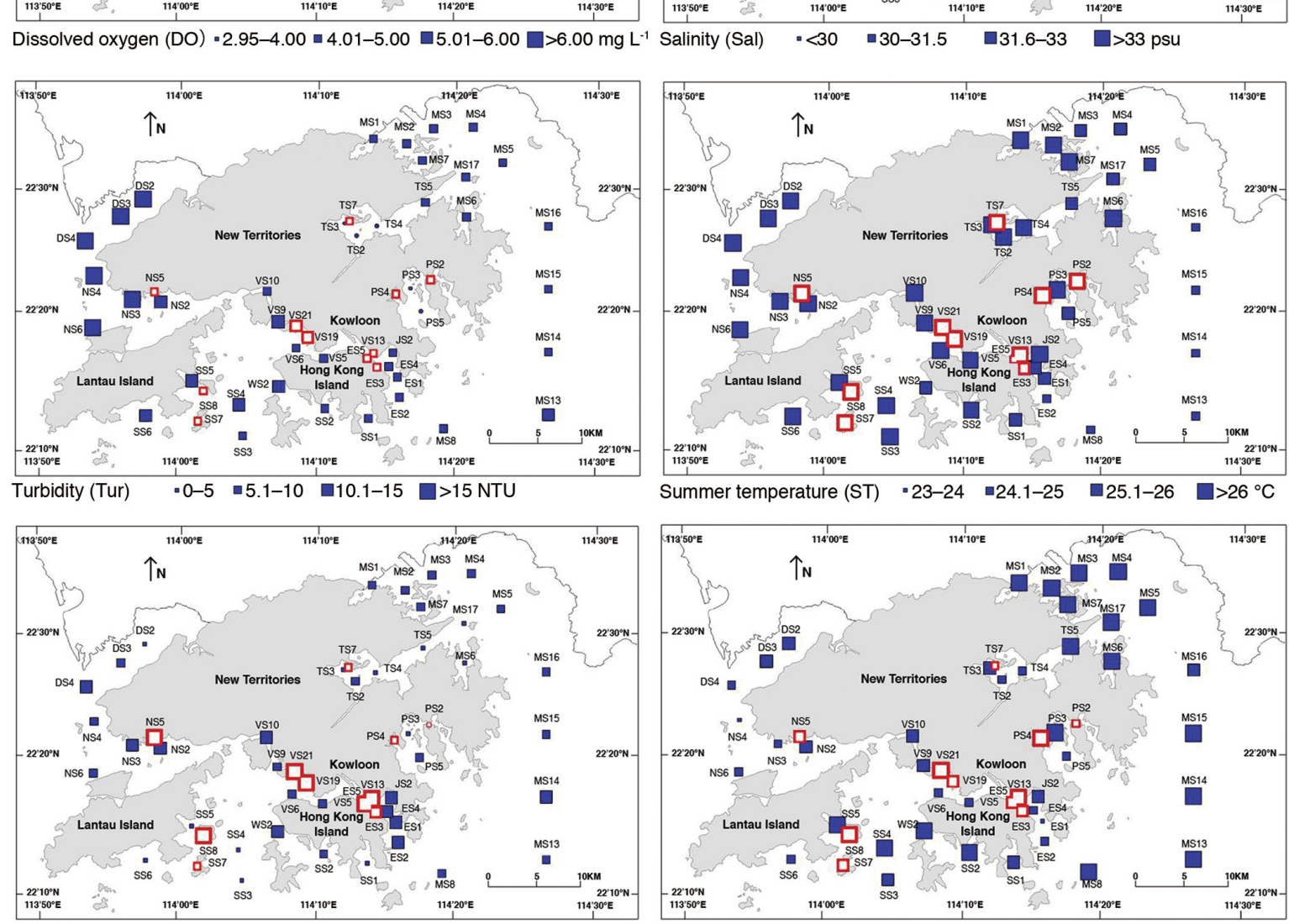

Winter temperature $(\mathrm{WT})=<19.5 \quad \square 9.51-20.0 \square 20.01-20.5 \square>20.5^{\circ} \mathrm{C}$

Mud content (MD) -40-55 $₫ 55.1-70 \square 70.1-85 \square>85 \% \mathrm{w} / \mathrm{w}(<63 \mu \mathrm{m})$

Figure 2. Spatial distribution of environmental parameters in Hong Kong. Mean surface water chlorophyll $a$ concentration; water depth; summer (June to September) bottom-water dissolved oxygen content; mean bottom-water salinity; mean turbidity; mean summer (June to September) bottom-water temperature; mean winter (November to February) bottom-water temperature; and mean mud content. All are averaged values of the data obtained during 1986-2013 (Table 1). 
Table 1. Summary of marine water-sediment parameters.

\begin{tabular}{llll}
\hline Parameter (abbreviation) & Unit & Sampling depth-material & Season \\
\hline Chlorophyll $a(\mathrm{Chl})$ & $\mu \mathrm{g} \mathrm{L}^{-1}$ & Surface water & All year \\
Water depth (D) & $\mathrm{m}$ & & All year \\
Dissolved oxygen (DO) & $\mathrm{mg} \mathrm{L}^{-1}$ & Bottom water & Summer \\
Mud content (MD) & $\% \mathrm{w} / \mathrm{w}(<63 \mu \mathrm{m})$ & Bottom sediment & All year \\
Salinity (Sal) & & Bottom water & All year \\
Turbidity (Tur) & $\mathrm{NTU}$ & Bottom water & All year \\
Summer temperature (ST) & ${ }^{\circ} \mathrm{C}$ & Bottom water & Summer \\
Winter temperature (WT) & ${ }^{\circ} \mathrm{C}$ & Bottom water & Winter \\
Copper $(\mathrm{Cu})$ & $\mathrm{mg} \mathrm{kg}$ & Bottom sediment & All year \\
Lead (Pb) & $\mathrm{mg} \mathrm{kg}^{-1}$ & Bottom sediment & All year \\
Zinc $(\mathrm{Zn})$ & $\mathrm{mg} \mathrm{kg}^{-1}$ & Bottom sediment & All year \\
\hline
\end{tabular}

${ }^{1}$ Summer: June, July, August, and September. ${ }^{2}$ Winter: November, December, January, and February.

\section{Materials and methods}

\subsection{Samples and laboratory procedure}

In January and July 2011 we used a Van Veen Grab to collect $100 \mathrm{~mL}$ of sediment from the uppermost centimeter of the seafloor from 52 sites in Hong Kong marine waters (Fig. 1; Supplement S1), 41 of which are in open waters and 11 in typhoon shelter sites. Typhoon shelters are semienclosed areas of water designed to protect moored vessels in extreme weather (Environmental Protection Department of Hong Kong, 2011). All sites are included in the Hong Kong EPD marine water and sediment quality monitoring program, which has been conducted monthly (for water) and biannually since 1986 (for sediment), providing comprehensive environmental data for all stations (see below).

Sediments were wet-sieved over a $63 \mu \mathrm{m}$ mesh sieve and air-dried or oven-dried at $40^{\circ} \mathrm{C}$. The residue was dry-sieved over a $150 \mu \mathrm{m}$ mesh sieve, and ostracod specimens larger than $150 \mu \mathrm{m}$ were picked; smaller individuals are mostly early instar juveniles that are often not preserved (because their shells are usually thin and delicate) or difficult to identify (see Yasuhara et al., 2009, 2017, for more details). In samples containing fewer than 200 specimens, we picked all individuals. If there were more than 200 specimens, we picked ostracods from a split. We identified each counted specimen to species level when possible. We considered either an entire carapace or a single valve as one individual for counting.

\subsection{Environmental variables}

We selected parameters from the EPD monitoring program (Table 1) for our regression modeling (see below), including surface productivity (chlorophyll $a$, Chl; Fig. 2), water depth (D; Fig. 2), bottom-water dissolved oxygen (DO; Fig. 2), bottom-water salinity (Sal; Fig. 2), turbidity (Tur; Fig. 2), summer bottom-water temperature (ST - June to
September average; Fig. 2), winter bottom-water temperature (WT - November to Feburary average; Fig. 2), mud content (MD; Fig. 2), and heavy metal concentration $(\mathrm{Cu}, \mathrm{Zn}$, and $\mathrm{Pb}$; Fig. 3). These parameters are known to control ostracod faunal properties (Cronin, 2015; Cronin and Vann, 2003; Hazel, 1988; Ikeya and Shiozaki, 1993; Irizuki et al., 2005, 2015a, 2018; Ruiz et al., 2005; Yasuhara et al., 2007, 2012b). We used averages over the entire monitoring period (19862011) because the ostracods in this study were mostly dead shells, and thus the samples should be considered time averaged. Bottom-water DO is the average of the summer season (June-September) due to the likely importance of summer bottom-water oxygen depletion.

\section{Regression modeling}

Multiple linear regression modeling was used to determine the relationship between common ostracod species (relative abundance of each species) and environmental parameters (Supplement S2). All environmental parameters were log-transformed and zero centered. Salinity outliers (salinity <25: DS2 and DS3) and samples with low abundance ( $<50$ specimens: ES5, MS7, VS21, VS5, and VS6) were removed from the models.

The best-fitting models were selected based on Akaike's information criterion (AIC) for small sample size, in which the lower score indicates the better model support considering both goodness-of-fit and model complexity (Anderson and Burnham, 2002). Akaike weights were used to summarize proportional support for all candidate models (Anderson et al., 2000) (Table 2). We considered parameter estimates averaged over models proportional to the support that each model received (Anderson et al., 2000) (Table 3). This approach accounts for the uncertainty in model selection and thus leads to appropriately broader confidence intervals than obtained by relying only on the single best-supported model. The relative importance of various predictor variables was 

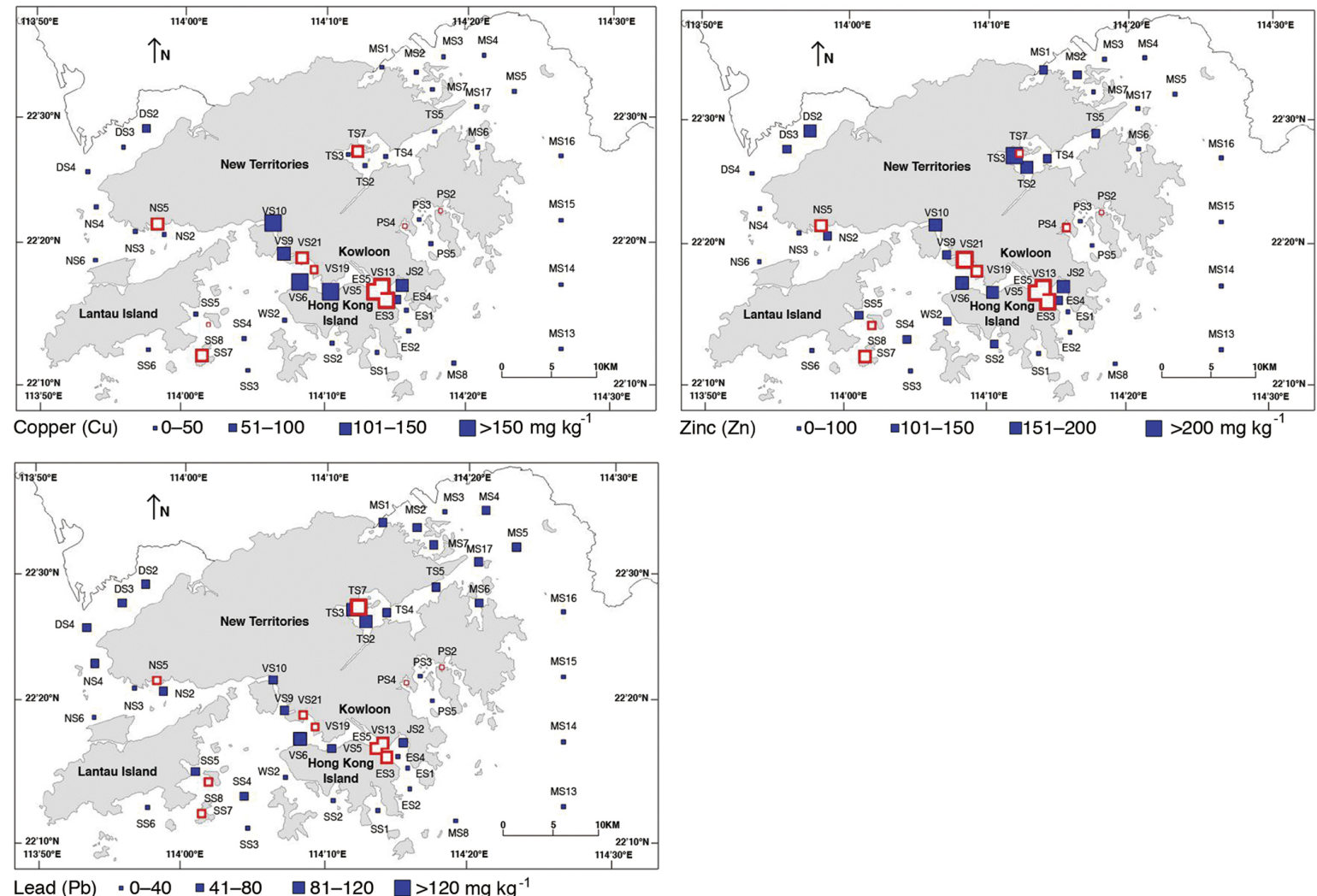

Figure 3. Spatial distribution of environmental parameters in Hong Kong. Mean copper $(\mathrm{Cu})$ concentration, mean lead $(\mathrm{Pb})$ concentration, and mean zinc (Zn) concentration in surface sediments. All are averaged values of the data obtained during 1986-2013 (Table 1).

measured by the sum of the Akaike weights of models that included the variables in question (Burnham and Anderson, 2002).

We explored linear dependencies by computing variance inflation factors (VIFs) (Legendre and Legendre, 1998) and pairwise correlations between predictor variables to assess whether multicollinearity was likely to influence regression results (Yasuhara et al., 2012b). The degree of freedom is more than one for the geographic region variable (see below), and thus we computed generalized variance inflation factors (GVIFs). For continuous variables, GVIF (Table 4) is the same as VIF, but for categorical variables, GVIF has degrees of freedom (DoF) equal to the number of coefficients associated with it (Hendrickx et al., 2004). Thus, we used $\mathrm{GVIF}^{1 / 2 \mathrm{DoF}}$ to make GVIF values comparable among those with different DoF. VIF $>20$ is usually indicative of high collinearity (Legendre and Legendre, 1998). Thus we calculated an equivalent threshold of 4.47 (equal to $\sqrt{ } 20$ ) for $\mathrm{GVIF}^{1 / 2 \mathrm{DoF}}$ to assess conlinearity. Also, adjusted $R^{2}>0.8$ indicates a strong correlation of variables (Hoffman, 2015). In all datasets, summer temperature (ST) and copper $(\mathrm{Cu})$ were highly correlated $\left(R^{2}=0.8217\right)$, and the GVIFs of ST and $\mathrm{Cu}$ are $>20$, indicating that these correlations may influence regression results. Thus, we re-ran the linear regression modeling without ST and $\mathrm{Cu}$. The new GVIFs of all variables were under 4.47 (Table 4).

We considered the degree of spatial autocorrelation in model residuals by the calculation of Moran's $I$ index for the five best models. The neighborhood size was set as 2,5 , 10,20 , and $50 \mathrm{~km}$. We found significant spatial autocorrelation in model residuals for many cases, and thus we forced the geographic region variable $(R)$ (water control zones defined by EPD) to be included in all models. After this treatment, spatial autocorrelation was detected only in a few models for Propontocypris spp., Stigmatocythere roesmani, and Hemikrithe orientalis.

The multiple linear regression model analyses were implemented in $\mathrm{R}$ programming language ( $\mathrm{R}$ Core Team, 2016). We used " $\mathrm{M}_{U} \mathrm{MI}_{N}$ " (Bartoń, 2013) for model averaging and "SPDEP" (Bivand and Piras, 2015) to measure spatial autocorrelation.

\section{Results and discussion}

The comprehensive ostracod dataset for the 52 sites and the environmental variables enabled us to elucidate distribution patterns of common ostracod taxa and their related environmental factor(s). We identified 151 species belong- 
Table 2. Best three regression models of the relative abundance of common species, including Pistocythereis bradyi, Bicornucythere bisanensis s.1., Nipponocythere bicarinata, Spinileberis quadriaculeata, Phlyctocythere japonica, Loxoconcha epeterseni, Sinocytheridea impressa, Neomonoceratina delicata, Keijella kloempritensis, Neosinocythere elongata, Stigmatocythere roesmani, Hemicytheridea reticulata, Loxoconcha malayensis, Alocopocythere goujoni, Hemikrithe orientalis, Propontocypris spp., Neonesidea spp., and Xestoleberis spp. The table shows the coefficient of each term, adjusted $R^{2}$, the Akaike information criterion corrected for small sample size (AIC), and the Akaike weight (AW). Bold denotes significance at $P<0.05$. Overall, $P$ is $<0.05$ in all models. R: region. Other abbreviations are found in Table 1 .

\begin{tabular}{|c|c|c|c|c|c|c|c|c|c|c|c|c|c|}
\hline Model & Chl & $\mathrm{D}$ & DO & MD & Sal & Tur & WT & $\mathrm{Pb}$ & $\mathrm{Zn}$ & $\mathrm{R}$ & $R^{2}$ & AIC & AW \\
\hline \multicolumn{14}{|c|}{ Pistocythereis bradyi } \\
\hline 1 & & -0.12 & & & 2.67 & & & -0.21 & & -0.06 & 0.61 & -163.64 & 0.52 \\
\hline 2 & & -0.13 & & & 2.87 & 0.04 & & -0.20 & & -0.05 & 0.61 & -159.79 & 0.08 \\
\hline 3 & & -0.12 & 0.07 & & 2.83 & & & -0.21 & & -0.07 & 0.60 & -159.42 & 0.06 \\
\hline \multicolumn{14}{|c|}{ Bicornucythere bisanensis s.l. } \\
\hline 1 & & -0.09 & & -0.23 & & & & & & 0.02 & 0.60 & -137.80 & 0.12 \\
\hline 2 & & -0.08 & & & & & & & & -0.01 & 0.56 & -136.77 & 0.07 \\
\hline 3 & & & & -0.29 & & & & 0.18 & & 0.00 & 0.58 & -136.77 & 0.05 \\
\hline \multicolumn{14}{|c|}{ Nipponocythere bicarinata } \\
\hline 1 & -0.07 & & -0.12 & & & & & & & 0.03 & 0.43 & -200.57 & 0.11 \\
\hline 2 & -0.09 & & & & & & & & & 0.03 & 0.39 & -200.38 & 0.10 \\
\hline 3 & -0.09 & -0.03 & -0.16 & & & & & & & 0.04 & 0.45 & -199.30 & 0.06 \\
\hline \multicolumn{14}{|c|}{ Spinileberis quadriaculeata } \\
\hline 1 & 0.06 & & & & & -0.09 & & & & -0.04 & 0.63 & -183.88 & 0.10 \\
\hline 2 & & -0.05 & & & & & & & & 0.02 & 0.60 & -182.94 & 0.06 \\
\hline 3 & 0.08 & & & & & -0.09 & -0.75 & & & -0.04 & 0.65 & -182.66 & 0.05 \\
\hline \multicolumn{14}{|c|}{ Phlyctocythere japonica } \\
\hline 1 & & 0.04 & & & & & & & & 0.00 & 0.28 & -238.60 & 0.19 \\
\hline 2 & & 0.03 & & & 0.33 & & & & & -0.02 & 0.30 & -237.01 & 0.09 \\
\hline 3 & & 0.03 & & & & & & -0.02 & & 0.00 & 0.27 & -235.62 & 0.04 \\
\hline \multicolumn{14}{|c|}{ Loxoconcha epeterseni } \\
\hline 1 & & 0.03 & & & -0.33 & -0.03 & & & & 0.01 & 0.49 & -288.61 & 0.11 \\
\hline 2 & 0.02 & 0.04 & & -0.04 & -0.34 & -0.04 & & & & 0.00 & 0.56 & -287.76 & 0.07 \\
\hline 3 & 0.02 & 0.04 & & & -0.34 & -0.04 & & & & 0.00 & 0.51 & -287.31 & 0.06 \\
\hline \multicolumn{14}{|c|}{ Sinocytheridea impressa } \\
\hline 1 & 0.33 & & -1.47 & 0.75 & -9.13 & & & & & 0.34 & 0.55 & -37.29 & 0.27 \\
\hline 2 & 0.41 & & -1.48 & & -8.75 & & & & & 0.37 & 0.50 & -35.24 & 0.10 \\
\hline 3 & & & -1.12 & 0.94 & -10.30 & & & & & 0.44 & 0.49 & -34.78 & 0.08 \\
\hline \multicolumn{14}{|c|}{ Neomonoceratina delicata } \\
\hline 1 & & & & & 3.77 & 0.34 & & & 0.41 & -0.13 & 0.48 & -82.57 & 0.29 \\
\hline 2 & & & 0.43 & & 5.02 & 0.31 & & & 0.39 & -0.20 & 0.50 & -81.21 & 0.15 \\
\hline 3 & & & & & 3.47 & 0.33 & & -0.20 & 0.55 & -0.12 & 0.48 & -79.32 & 0.06 \\
\hline \multicolumn{14}{|c|}{ Keijella kloempritensis } \\
\hline 1 & & 0.06 & & & & & & -0.06 & & -0.02 & 0.56 & -202.12 & 0.12 \\
\hline 2 & & 0.08 & & & & & & & & -0.02 & 0.53 & -201.51 & 0.09 \\
\hline 3 & & 0.06 & & 0.09 & & & & -0.08 & & -0.02 & 0.58 & -200.86 & 0.06 \\
\hline
\end{tabular}


Table 2. Continued.

\begin{tabular}{|c|c|c|c|c|c|c|c|c|c|c|c|c|c|}
\hline Model & Chl & $\mathrm{D}$ & $\mathrm{DO}$ & $\mathrm{MD}$ & Sal & Tur & WT & $\mathrm{Pb}$ & $\mathrm{Zn}$ & $\mathrm{R}$ & $R^{2}$ & AIC & AW \\
\hline \multicolumn{14}{|c|}{ Neosinocythere elongata } \\
\hline 1 & -0.02 & & & & & 0.09 & & & & -0.03 & 0.76 & -244.70 & 0.09 \\
\hline 2 & & & & -0.06 & & 0.08 & & & & -0.03 & 0.76 & -244.42 & 0.08 \\
\hline 3 & & & & & & 0.09 & & & & -0.03 & 0.74 & -244.15 & 0.07 \\
\hline \multicolumn{14}{|c|}{ Stigmatocythere roesmani } \\
\hline 1 & & & & & & & & -0.08 & & 0.00 & 0.23 & -225.23 & 0.07 \\
\hline 2 & & & & & 0.36 & & & $-\mathbf{0 . 0 7}$ & & -0.02 & 0.25 & -224.06 & 0.04 \\
\hline 3 & & & 0.10 & & 0.67 & & & -0.07 & & $-\mathbf{0 . 0 3}$ & 0.30 & -224.03 & 0.04 \\
\hline \multicolumn{14}{|c|}{ Hemicytheridea reticulata } \\
\hline 1 & & $-\mathbf{0 . 0 3}$ & & & & & & -0.04 & & 0.01 & 0.23 & -270.79 & 0.14 \\
\hline 2 & 0.02 & -0.02 & & & & & & -0.04 & & 0.01 & 0.26 & -269.44 & 0.07 \\
\hline 3 & $\mathbf{0 . 0 3}$ & & & & & & & $-\mathbf{0 . 0 3}$ & & 0.00 & 0.19 & -268.64 & 0.05 \\
\hline \multicolumn{14}{|c|}{ Loxoconcha malayensis } \\
\hline 1 & & -0.03 & -0.14 & -0.12 & & & & & & 0.03 & 0.55 & -229.55 & 0.10 \\
\hline 2 & 0.04 & & -0.13 & -0.12 & & & & & & 0.02 & 0.55 & -229.42 & 0.09 \\
\hline 3 & & -0.04 & -0.14 & -0.10 & & & & -0.04 & & 0.03 & 0.57 & -228.47 & 0.06 \\
\hline \multicolumn{14}{|c|}{ Alocopocythere goujoni } \\
\hline 1 & & & & & 0.21 & & & & & -0.04 & 0.68 & -331.97 & 0.09 \\
\hline 2 & & -0.01 & & & 0.28 & & & & -0.01 & -0.04 & 0.71 & -330.52 & 0.05 \\
\hline 3 & & -0.01 & & & 0.27 & & & -0.02 & & -0.04 & 0.71 & -330.45 & 0.04 \\
\hline \multicolumn{14}{|c|}{ Hemikrithe orientalis } \\
\hline 1 & & 0.03 & & & & & 0.46 & & & 0.00 & 0.19 & -249.44 & 0.11 \\
\hline 2 & & 0.03 & 0.07 & & & & & & & 0.00 & 0.18 & -248.67 & 0.07 \\
\hline 3 & & 0.04 & 0.06 & & & & 0.42 & & & 0.00 & 0.23 & -248.60 & 0.07 \\
\hline \multicolumn{14}{|c|}{ Propontocypris spp. } \\
\hline 1 & -0.28 & & & 0.46 & & & & & & 0.04 & 0.20 & -72.47 & 0.15 \\
\hline 2 & -0.24 & & & & & & & & & 0.07 & 0.13 & -71.74 & 0.10 \\
\hline 3 & -0.24 & & & 0.49 & & & -2.46 & & & 0.02 & 0.22 & -70.76 & 0.06 \\
\hline \multicolumn{14}{|c|}{ Neonesidea spp. } \\
\hline 1 & -0.12 & & & -0.25 & & & & & 0.19 & 0.05 & 0.28 & -149.00 & 0.09 \\
\hline 2 & -0.11 & & 0.33 & -0.24 & & & -1.13 & & 0.20 & 0.05 & 0.31 & -147.86 & 0.05 \\
\hline 3 & & & 0.21 & & & & & & & 0.02 & 0.08 & -147.40 & 0.04 \\
\hline \multicolumn{14}{|c|}{ Xestoleberis spp. } \\
\hline 1 & & 0.04 & 0.15 & -0.11 & & -0.08 & & 0.05 & & -0.02 & 0.62 & -225.55 & 0.09 \\
\hline 2 & & 0.03 & 0.15 & -0.09 & & -0.09 & & & & -0.02 & 0.59 & -225.29 & 0.08 \\
\hline 3 & & & & -0.09 & & -0.06 & & & & 0.00 & 0.52 & -224.60 & 0.06 \\
\hline
\end{tabular}

ing to 76 genera (Supplement S1). Among them, 18 common taxa (mainly species, a few genera) of Pistocythereis bradyi, Bicornucythere bisanensis s.1., Nipponocythere bicarinata, Spinileberis quadriaculeata, Phlyctocythere japonica, Loxoconcha epeterseni, Sinocytheridea impressa, Neomonoceratina delicata, Keijella kloempritensis, Neosinocythere elongata, Stigmatocythere roesmani, Hemicytheridea retic- ulata Loxoconcha malayensis, Alocopocythere goujoni, Hemikrithe orientalis, Propontocypris spp., Neonesidea spp., and Xestoleberis spp. (Supplement S2) were used for regression modeling, and their relative abundances (to the total ostracod abundance in a sample) show significant relations to environmental parameters. The best three regression models are presented in Table 2 and the model-averaged parameter 
Table 3. Model-averaged parameter estimates and CIs of the relative abundance for common species including Pistocythereis bradyi, Bicornucythere bisanensis s.1., Nipponocythere bicarinata, Spinileberis quadriaculeata, Phlyctocythere japonica, Loxoconcha epeterseni, Sinocytheridea impressa, Neomonoceratina delicata, Keijella kloempritensis, Neosinocythere elongata, Stigmatocythere roesmani, Hemicytheridea reticulata Loxoconcha malayensis, Alocopocythere goujoni, Hemikrithe orientalis, Propontocypris spp., Neonesidea spp., and Xestoleberis spp. CIs, confidence intervals; RI, relative importance (the sum of the Akaike weights of models that include the variable in question; see Materials and Methods); R, region. Other abbreviations are found in Table 1. Bold denotes CIs that exclude zero. For R, coefficient, lower CI, and upper CI values shown are averages of those for geographic regions.

\begin{tabular}{|c|c|c|c|c|c|c|c|c|c|}
\hline Term & RI & Coefficient & Lower CI & Upper CI & Term & RI & Coefficient & Lower CI & Upper CI \\
\hline \multicolumn{5}{|c|}{ Pistocythereis bradyi } & \multicolumn{5}{|c|}{ Bicornucythere bisanensis s.1. } \\
\hline $\mathrm{R}$ & 1.00 & -0.06 & -0.15 & 0.04 & $\mathrm{R}$ & 1.00 & 0.01 & -0.11 & 0.12 \\
\hline Sal & 1.00 & 2.71 & 1.53 & 3.88 & MD & 0.63 & -0.26 & -0.52 & 0.00 \\
\hline D & 1.00 & -0.12 & -0.19 & -0.06 & D & 0.62 & -0.08 & -0.16 & 0.00 \\
\hline $\mathbf{P b}$ & 0.97 & -0.21 & -0.32 & -0.10 & $\mathrm{~Pb}$ & 0.38 & 0.14 & -0.05 & 0.33 \\
\hline Tur & 0.12 & 0.04 & -0.07 & 0.15 & DO & 0.20 & 0.17 & -0.15 & 0.48 \\
\hline $\mathrm{Zn}$ & 0.11 & -0.05 & -0.23 & 0.13 & Chl & 0.19 & 0.06 & -0.07 & 0.19 \\
\hline DO & 0.10 & 0.06 & -0.18 & 0.31 & Tur & 0.19 & 0.07 & -0.08 & 0.22 \\
\hline WT & 0.10 & 0.33 & -0.98 & 1.64 & $\mathrm{Zn}$ & 0.16 & -0.01 & -0.28 & 0.25 \\
\hline Chl & 0.09 & 0.02 & -0.08 & 0.11 & Sal & 0.12 & -0.02 & -1.86 & 1.82 \\
\hline MD & 0.08 & -0.01 & -0.20 & 0.18 & WT & 0.10 & 0.06 & -1.80 & 1.92 \\
\hline \multicolumn{5}{|c|}{ Nipponocythere bicarinata } & \multicolumn{5}{|c|}{ Spinileberis quadriaculeata } \\
\hline $\mathrm{R}$ & 1.00 & 0.02 & -0.04 & 0.09 & $\mathrm{R}$ & & -0.01 & -0.10 & 0.07 \\
\hline Chl & 0.92 & -0.08 & -0.14 & -0.02 & Tur & 0.66 & -0.09 & -0.17 & 0.00 \\
\hline DO & 0.50 & -0.14 & -0.30 & 0.02 & Chl & 0.54 & 0.06 & 0.00 & 0.13 \\
\hline $\mathrm{D}$ & 0.30 & -0.03 & -0.08 & 0.02 & $\mathrm{D}$ & 0.45 & -0.05 & -0.10 & 0.01 \\
\hline WT & 0.25 & -0.03 & -0.08 & 0.02 & WT & 0.32 & -0.76 & -1.82 & 0.31 \\
\hline Sal & 0.24 & 0.51 & -0.35 & 1.36 & DO & 0.19 & 0.10 & -0.10 & 0.30 \\
\hline Tur & 0.19 & -0.04 & -0.11 & 0.04 & $\mathrm{MD}$ & 0.17 & -0.07 & -0.23 & 0.09 \\
\hline $\mathrm{Zn}$ & 0.18 & -0.04 & -0.12 & 0.04 & $\mathrm{~Pb}$ & 0.17 & -0.05 & -0.16 & 0.07 \\
\hline $\mathrm{Pb}$ & 0.13 & -0.03 & -0.11 & 0.06 & $\mathrm{Zn}$ & 0.14 & 0.03 & -0.09 & 0.15 \\
\hline MD & 0.10 & 0.00 & -0.13 & 0.13 & Sal & 0.13 & 0.11 & -1.03 & 1.26 \\
\hline \multicolumn{5}{|c|}{ Phlyctocythere japonica } & \multicolumn{5}{|c|}{ Loxoconcha epeterseni } \\
\hline $\mathrm{R}$ & 1.00 & -0.01 & -0.05 & 0.03 & $\mathrm{R}$ & 1.00 & 0.00 & -0.02 & 0.03 \\
\hline D & $\mathbf{0 . 8 0}$ & 0.03 & 0.01 & 0.06 & D & 0.94 & 0.03 & 0.00 & 0.05 \\
\hline Sal & 0.42 & 0.46 & -0.10 & 1.02 & Tur & 0.76 & -0.03 & -0.06 & 0.00 \\
\hline $\mathrm{Pb}$ & 0.21 & -0.03 & -0.08 & 0.03 & Sal & 0.53 & -0.30 & -0.62 & 0.02 \\
\hline $\mathrm{Zn}$ & 0.20 & -0.02 & -0.07 & 0.03 & $\mathrm{MD}$ & 0.38 & -0.04 & -0.08 & 0.01 \\
\hline WT & 0.15 & 0.22 & -0.38 & 0.81 & Chl & 0.30 & 0.02 & -0.01 & 0.04 \\
\hline DO & 0.14 & 0.03 & -0.08 & 0.15 & DO & 0.26 & 0.04 & -0.02 & 0.10 \\
\hline Tur & 0.12 & 0.00 & -0.05 & 0.05 & $\mathrm{~Pb}$ & 0.17 & 0.01 & -0.02 & 0.05 \\
\hline Chl & 0.12 & 0.00 & -0.04 & 0.05 & WT & 0.13 & -0.13 & -0.48 & 0.22 \\
\hline MD & 0.11 & 0.00 & -0.09 & 0.08 & $\mathrm{Zn}$ & 0.12 & 0.01 & -0.03 & 0.04 \\
\hline \multicolumn{5}{|c|}{ Sinocytheridea impressa } & \multicolumn{5}{|c|}{ Neomonoceratina delicata } \\
\hline $\mathrm{R}$ & 1.00 & 0.36 & -0.09 & 0.81 & $\mathrm{R}$ & 1.00 & -0.15 & -0.42 & 0.12 \\
\hline Sal & 0.99 & -9.26 & -14.61 & -3.91 & $\mathbf{Z n}$ & 0.94 & 0.41 & 0.11 & 0.70 \\
\hline DO & 0.91 & -1.42 & -2.48 & $-\mathbf{0 . 3 7}$ & Sal & 0.93 & 4.06 & 1.00 & 7.13 \\
\hline MD & 0.75 & 0.82 & 0.07 & 1.57 & Tur & 0.84 & 0.33 & 0.06 & 0.59 \\
\hline Chl & 0.74 & 0.82 & 0.03 & 0.70 & DO & 0.33 & 0.45 & -0.17 & 1.06 \\
\hline WT & 0.20 & 0.82 & -2.34 & 8.89 & $\mathrm{~Pb}$ & 0.17 & -0.18 & -0.67 & 0.30 \\
\hline $\mathrm{Zn}$ & 0.13 & 0.82 & -0.68 & 0.51 & $\mathrm{MD}$ & 0.14 & 0.25 & -0.33 & 0.84 \\
\hline Tur & 0.11 & 0.82 & -0.61 & 0.30 & $\mathrm{D}$ & 0.11 & -0.01 & -0.22 & 0.20 \\
\hline $\mathrm{D}$ & 0.11 & 0.82 & -0.29 & 0.41 & WT & 0.11 & -0.86 & -4.32 & 2.60 \\
\hline $\mathrm{Pb}$ & 0.10 & 0.82 & -0.62 & 0.46 & Chl & 0.09 & 0.00 & -0.24 & 0.23 \\
\hline
\end{tabular}


Table 3. Continued.

\begin{tabular}{|c|c|c|c|c|c|c|c|c|c|}
\hline Term & RI & Coefficient & Lower CI & Upper CI & Term & RI & Coefficient & Lower CI & Upper CI \\
\hline \multicolumn{5}{|c|}{ Keijella kloempritensis } & \multicolumn{5}{|c|}{ Neosinocythere elongata } \\
\hline $\mathrm{R}$ & 1.00 & -0.02 & -0.09 & 0.04 & $\mathrm{R}$ & 1.00 & -0.03 & -0.07 & 0.00 \\
\hline D & 0.86 & 0.06 & 0.02 & 0.11 & Tur & 1.00 & 0.08 & 0.04 & 0.13 \\
\hline $\mathrm{Pb}$ & 0.58 & -0.08 & -0.17 & 0.01 & MD & 0.33 & -0.05 & -0.13 & 0.02 \\
\hline Sal & 0.35 & 0.68 & -0.21 & 1.58 & Chl & 0.30 & -0.02 & -0.05 & 0.01 \\
\hline MD & 0.25 & 0.08 & -0.05 & 0.21 & WT & 0.26 & -0.33 & -0.85 & 0.18 \\
\hline Tur & 0.24 & 0.05 & -0.03 & 0.14 & $\mathrm{Zn}$ & 0.21 & -0.02 & -0.06 & 0.02 \\
\hline Chl & 0.22 & -0.04 & -0.11 & 0.03 & $\mathrm{~Pb}$ & 0.19 & -0.02 & -0.07 & 0.02 \\
\hline $\mathrm{Zn}$ & 0.17 & 0.02 & -0.11 & 0.14 & $\mathrm{D}$ & 0.12 & 0.00 & -0.03 & 0.03 \\
\hline WT & 0.14 & 0.33 & -0.55 & 1.22 & DO & 0.12 & -0.02 & -0.11 & 0.07 \\
\hline DO & 0.11 & 0.03 & -0.14 & 0.19 & Sal & 0.10 & 0.04 & -0.40 & 0.49 \\
\hline \multicolumn{5}{|c|}{ Stigmatocythere roesmani } & \multicolumn{5}{|c|}{ Hemicytheridea reticulata } \\
\hline $\mathrm{R}$ & 1.00 & -0.01 & -0.07 & 0.05 & $\mathrm{R}$ & 1.00 & 0.01 & -0.02 & 0.03 \\
\hline Sal & 0.61 & 0.62 & -0.04 & 1.27 & $\mathbf{P b}$ & 0.67 & -0.04 & -0.07 & 0.00 \\
\hline $\mathbf{P b}$ & 0.61 & -0.06 & -0.12 & 0.00 & D & 0.66 & -0.02 & -0.04 & 0.00 \\
\hline Tur & 0.37 & 0.04 & -0.01 & 0.10 & Chl & 0.49 & 0.03 & 0.00 & 0.05 \\
\hline $\mathrm{Zn}$ & 0.31 & -0.05 & -0.11 & 0.02 & WT & 0.24 & -0.24 & -0.64 & 0.16 \\
\hline DO & 0.31 & 0.09 & -0.04 & 0.22 & MD & 0.23 & -0.03 & -0.09 & 0.02 \\
\hline MD & 0.23 & -0.06 & -0.16 & 0.04 & $\mathrm{Zn}$ & 0.17 & -0.01 & -0.06 & 0.05 \\
\hline Chl & 0.19 & -0.02 & -0.07 & 0.02 & DO & 0.13 & 0.02 & -0.05 & 0.10 \\
\hline WT & 0.19 & 0.35 & -0.33 & 1.02 & Tur & 0.13 & -0.01 & -0.05 & 0.03 \\
\hline $\mathrm{D}$ & 0.14 & -0.01 & -0.05 & 0.04 & Sal & 0.11 & -0.05 & -0.43 & 0.34 \\
\hline \multicolumn{5}{|c|}{ Loxoconcha malayensis } & \multicolumn{5}{|c|}{ Alocopocythere goujoni } \\
\hline $\mathrm{R}$ & 1.00 & 0.02 & -0.02 & 0.06 & $\mathrm{R}$ & 1.00 & -0.03 & -0.05 & -0.02 \\
\hline DO & 0.87 & -0.14 & -0.25 & -0.03 & Sal & 0.78 & 0.22 & 0.03 & 0.41 \\
\hline MD & 0.85 & -0.11 & -0.20 & -0.02 & $\mathrm{D}$ & 0.46 & -0.01 & -0.02 & 0.00 \\
\hline $\mathrm{D}$ & 0.48 & -0.03 & -0.06 & 0.00 & DO & 0.43 & -0.03 & -0.07 & 0.01 \\
\hline Chl & 0.40 & 0.03 & -0.01 & 0.08 & $\mathrm{~Pb}$ & 0.31 & -0.01 & -0.03 & 0.01 \\
\hline $\mathrm{Pb}$ & 0.29 & -0.04 & -0.10 & 0.02 & $\mathrm{Zn}$ & 0.29 & -0.01 & -0.03 & 0.01 \\
\hline WT & 0.26 & 0.41 & -0.21 & 1.03 & WT & 0.24 & 0.13 & -0.08 & 0.33 \\
\hline $\mathrm{Zn}$ & 0.20 & -0.03 & -0.10 & 0.04 & Tur & 0.18 & 0.01 & -0.01 & 0.03 \\
\hline Sal & 0.15 & -0.26 & -0.91 & 0.39 & MD & 0.14 & -0.01 & -0.04 & 0.02 \\
\hline Tur & 0.11 & -0.01 & -0.07 & 0.05 & Chl & 0.11 & 0.00 & -0.01 & 0.01 \\
\hline \multicolumn{5}{|c|}{ Hemikrithe orientalis } & \multicolumn{5}{|c|}{ Propontocypris spp. } \\
\hline $\mathrm{R}$ & 1.00 & 0.00 & -0.04 & 0.03 & $\mathrm{R}$ & 1.00 & 0.05 & -0.19 & 0.30 \\
\hline D & 0.77 & 0.03 & 0.00 & 0.05 & Chl & 0.80 & -0.26 & -0.47 & -0.04 \\
\hline WT & 0.46 & 0.43 & -0.07 & 0.93 & $\mathrm{MD}$ & 0.58 & 0.50 & -0.03 & 1.03 \\
\hline DO & 0.44 & 0.08 & -0.02 & 0.17 & WT & 0.29 & -2.60 & -6.48 & 1.28 \\
\hline Sal & 0.30 & 0.37 & -0.17 & 0.90 & $\mathrm{~Pb}$ & 0.19 & -0.18 & -0.55 & 0.20 \\
\hline $\mathrm{Pb}$ & 0.22 & -0.03 & -0.08 & 0.02 & Sal & 0.17 & -1.48 & -4.88 & 1.92 \\
\hline Tur & 0.17 & 0.02 & -0.03 & 0.07 & DO & 0.15 & -0.27 & -0.99 & 0.45 \\
\hline Chl & 0.14 & -0.01 & -0.05 & 0.03 & $\mathrm{Zn}$ & 0.14 & -0.03 & -0.48 & 0.41 \\
\hline $\mathrm{Zn}$ & 0.11 & 0.00 & -0.05 & 0.05 & $\mathrm{D}$ & 0.13 & 0.04 & -0.17 & 0.26 \\
\hline MD & 0.10 & -0.01 & -0.08 & 0.07 & Tur & 0.10 & 0.01 & -0.29 & 0.32 \\
\hline
\end{tabular}


Table 3. Continued.

\begin{tabular}{|c|c|c|c|c|c|c|c|c|c|}
\hline Term & RI & Coefficient & Lower CI & Upper CI & Term & RI & Coefficient & Lower CI & Upper CI \\
\hline \multicolumn{5}{|c|}{ Neonesidea spp. } & \multicolumn{5}{|c|}{ Xestoleberis spp. } \\
\hline $\mathrm{R}$ & 1.00 & 0.02 & -0.09 & 0.13 & $\mathrm{R}$ & 1.00 & -0.01 & -0.06 & 0.05 \\
\hline DO & 0.72 & 0.30 & 0.02 & 0.57 & Tur & 0.77 & $-\mathbf{0 . 0 7}$ & -0.12 & -0.01 \\
\hline $\mathrm{Zn}$ & 0.58 & 0.15 & -0.01 & 0.31 & MD & 0.66 & $-\mathbf{0 . 1 0}$ & -0.19 & 0.00 \\
\hline MD & 0.46 & -0.23 & -0.49 & 0.03 & DO & 0.63 & 0.13 & 0.00 & 0.26 \\
\hline Chl & 0.42 & -0.09 & -0.21 & 0.02 & $\mathrm{D}$ & 0.42 & 0.03 & -0.01 & 0.07 \\
\hline WT & 0.32 & -1.13 & -2.69 & 0.42 & $\mathrm{~Pb}$ & 0.33 & 0.04 & -0.02 & 0.11 \\
\hline Tur & 0.24 & -0.09 & -0.22 & 0.05 & Sal & 0.27 & 0.46 & -0.24 & 1.15 \\
\hline $\mathrm{D}$ & 0.23 & 0.05 & -0.04 & 0.15 & WT & 0.19 & -0.37 & -1.06 & 0.31 \\
\hline $\mathrm{Pb}$ & 0.17 & 0.05 & -0.13 & 0.24 & $\mathrm{Zn}$ & 0.14 & 0.00 & -0.09 & 0.08 \\
\hline Sal & 0.15 & -0.60 & -2.20 & 1.01 & Chl & 0.10 & 0.00 & -0.05 & 0.05 \\
\hline
\end{tabular}

Table 4. GVIF value for environmental variables. DoF, degrees of freedom; R, region. Other abbreviations are found in Table 1.

\begin{tabular}{lrrr}
\hline $\begin{array}{l}\text { Environmental } \\
\text { variables }\end{array}$ & GVIF & DoF & GVIF $^{1 / 2 D o F}$ \\
\hline Chl & 7.40 & 1 & 2.72 \\
D & 6.41 & 1 & 2.53 \\
DO & 4.49 & 1 & 2.12 \\
MD & 3.72 & 1 & 1.93 \\
Sal & 9.43 & 1 & 3.07 \\
Tur & 6.67 & 1 & 2.58 \\
Pb & 9.98 & 1 & 3.16 \\
Zn & 10.05 & 1 & 3.17 \\
WT & 2.39 & 1 & 1.55 \\
R & 1266.30 & 9 & 1.49 \\
\hline
\end{tabular}

estimates in Table 3. A small percentage of specimens of phytal genera (e.g., Xestoleberis spp., Neonesidea spp.) were contained in each sample, which are basically allochthonous specimens in bottom sediments transported from surrounding phytal environments. The value of allochthonous species to environmental interpretation is limited; however, most ostracod specimens in each sample are composed of benthic muddy sediment dwellers, which are considered autochthonous.

Ostracods were divided into four groups based on their geographic distributions: (a) widespread group; (b) temperate group; (c) subtropical group; (d) tropical group; and (e) globally distributed group (Fig. 4).

\subsection{Widespread group}

The widespread group constitutes only one species, Pistocythereis bradyi. Pistocythereis bradyi is widely distributed throughout the marginal marine environments of Japan, the East and South China Sea, and the Indo-Pacific (Fig. 4). Relative abundance of $P$. bradyi was highly correlated with salinity (positive), water depth (negative), and $\mathrm{Pb}$ (negative) (Ta- bles 2 and 3). This indicates that the species prefers relatively shallow environments with high salinity (Figs. 2 and 5). In the Pearl River Delta and shallow South China Sea (Fig. 4), $P$. bradyi is dominant along the inner continental shelf at water depths $<100 \mathrm{~m}$ (mostly common between 10 and $50 \mathrm{~m}$ ) and at salinities from 30-40 (Li, 1985; Zhao and Wang, 1990; Zhao et al., 1986). Pistocythereis bradyi is a typical middle muddy bay species in Japan (Irizuki et al., 2006; Yasuhara and Irizuki, 2001; Yasuhara and Seto, 2006), and known from open bays such as Gamagyang Bay in Korea (Abe, 1988) and Malacca Strait (Whatley and Zhao, 1988b). In these studies, $P$. bradyi prefers relatively high salinity and deeper water in the inner continental shelf. Our data agree as to the preference for high salinity, but are inconsistent with the literature regarding shallower water depths (Tables 2 and 3). Salinity may be more important than depth, but the restricted depth range of our sites $(\leq 35 \mathrm{~m})$ may also be a reason for this inconsistency. Our results indicate that $P$. bradyi is sensitive to metal pollution (Tables 2 and 3).

\subsection{Temperate group}

Five species including Bicornucythere bisanensis s.1., Nipponocythere bicarinata, Spinileberis quadriaculeata, Phlyctocythere japonica, and Loxoconcha epeterseni are distributed from the Japanese-Russian coast to the South China Sea.

Bicornucythere bisanensis s.l. is very common in most samples, the relative abundance of which was significantly correlated with water depth (negative) (Tables 2 and 3), and this species prefers shallower environments (Figs. 2 and 5). In Chinese and Japanese coastal areas, B. bisanensis s.l. is abundant in brackish water (salinity: 20-30) at depths less than $10 \mathrm{~m}$ (Ikeya and Shiozaki, 1993; Irizuki et al., 2006; Zhao et al., 1986). Our results confirm this preference for shallow depths. In Japan, Bicornucythere bisanensis is tolerant of anthropogenic impacts, especially eutrophication and the resulting bottom-water hypoxia (Irizuki et al., 2003, 2011, 2015a, 2018; Yasuhara et al., 2003, 2007, 


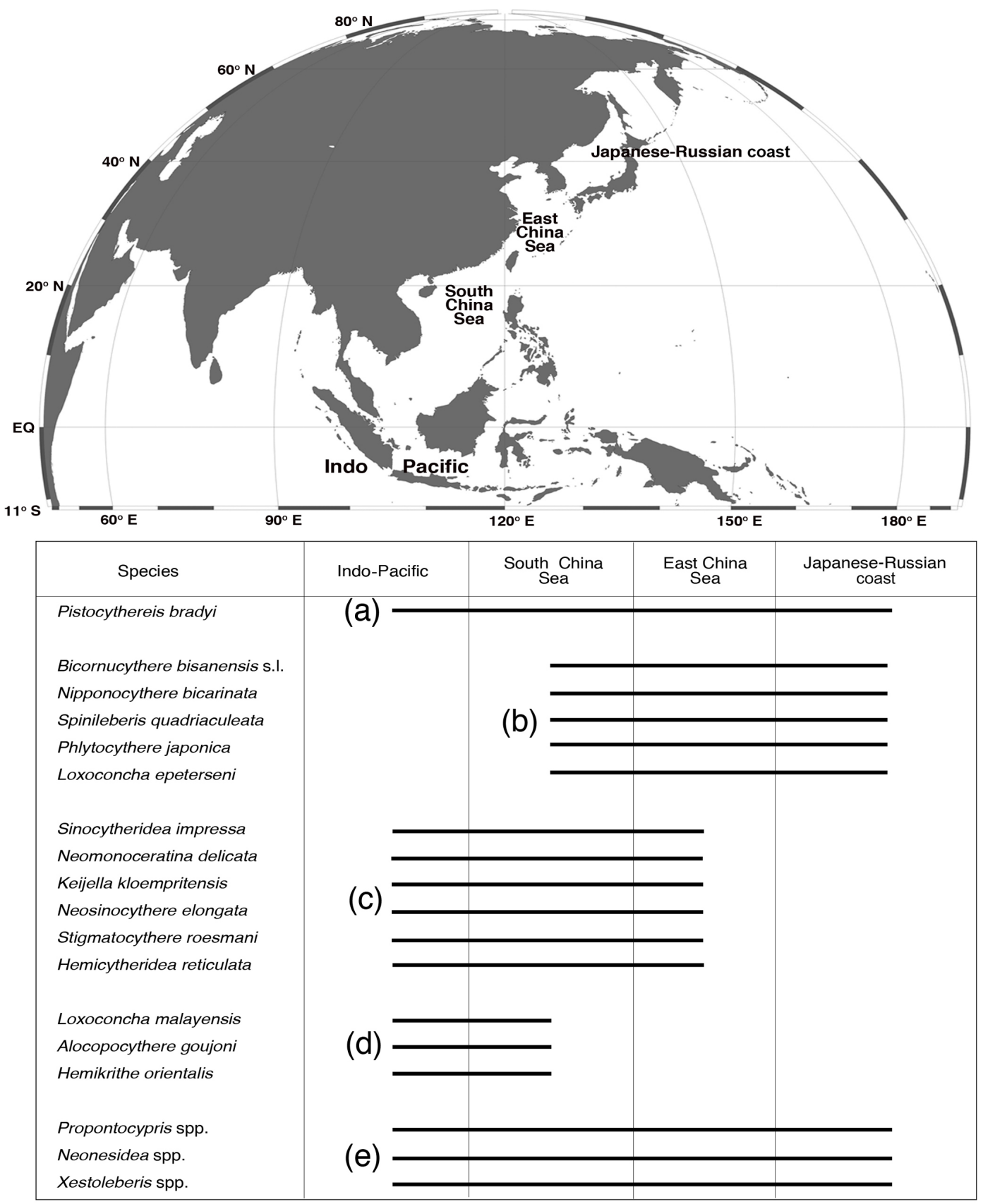

Figure 4. Geographical distributions of the 18 taxa in the northwestern Pacific and Indo-Pacific regions, including Pistocythereis bradyi, Bicornucythere bisanensis s.1., Nipponocythere bicarinata, Spinileberis quadriaculeata, Phlyctocythere japonica, Loxoconcha epeterseni, Sinocytheridea impressa, Neomonoceratina delicata, Keijella kloempritensis, Neosinocythere elongata, Stigmatocythere roesmani, Hemicytheridea reticulata Loxoconcha malayensis, Alocopocythere goujoni, Hemikrithe orientalis, Propontocypris spp., Neonesidea spp., and Xestoleberis spp. (a) Widespread group; (b) temperate group; (c) subtropical group; (d) tropical group; and (e) globally distributed group. The following references were used mainly to determine the geographical distributions of the species: Al Jumaily and Al-Sheikhly (1999); Dewi (1997); Dong et al. (2012); Fauzielly et al. (2013); Gu et al. (2017); Hong et al. (2017); Hou and Gou (2007); Hussain et al. (2004, 2010); Hussain and Mohan (2000, 2001); Irizuki et al. (2006, 2009); Iwatani et al. (2014); Liu et al. (2013); Li (1985); Mostafawi (1992); Nishath et al. (2017); Noraswana et al. (2014); Pugliese et al. (2006); Schornikov et al. (2014); Tanaka et al. (2009, 2011); Wang et al. (1988); Wang and Zhang (1987); Wang and Zhao (1985); Zhao (1984); Zhao and Wang (1988a, b, 1990); Zhao and Whatley (1993); Zhou et al. (2015). Note that Sinocytheridea impressa is known to be distributed in Japan, but their Japanese distribution is very limited in certain areas of the southern part of Japan (Iwasaki, 1992; Tanaka et al., 2019). Thus, we did not indicate their Japanese-Russian coast distribution in this figure. 

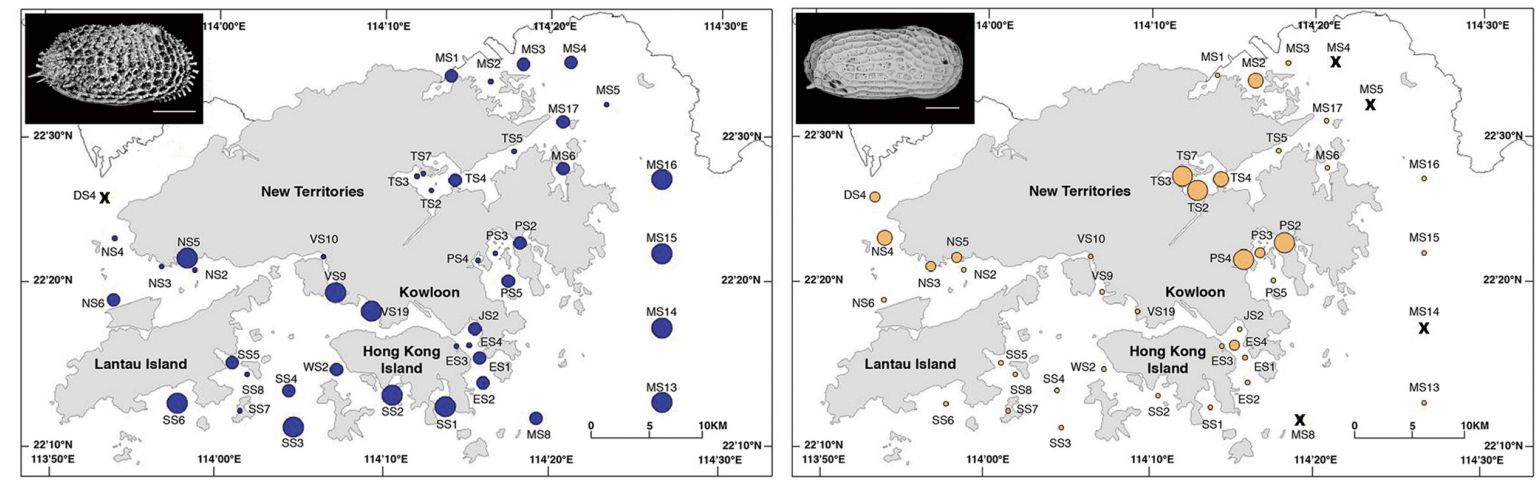

Pistocythereis bradyi $\quad 0<\bullet \leq 5 \% \quad 5<\bullet \leq 10 \% \quad 10<0 \leq 15 \% \quad x$ Absent

Bicornucythere bisanensis s.l. $\begin{aligned} & 0<0 \leq 5 \% \\ & 15<0<0\end{aligned} \quad \begin{aligned} & 5<0 \leq 10 \% \\ & \text { x Absent }\end{aligned}$
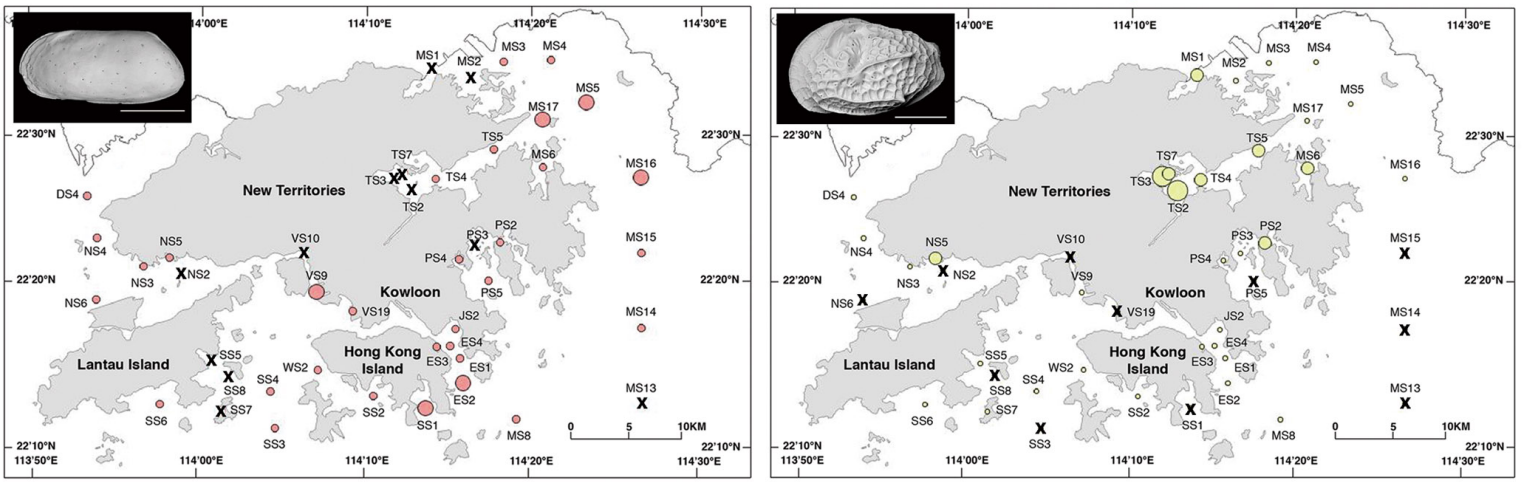

Nipponocythere bicarinata $\quad 0<0 \leq 5 \% \quad 5<0 \leq 10 \% \quad$ x Absent

Spinileberis quadriaculeata $\quad 0<0 \leq 5 \% \quad 5<0 \leq 10 \% \quad 10 \% \leq \bigcirc \times$ Absent
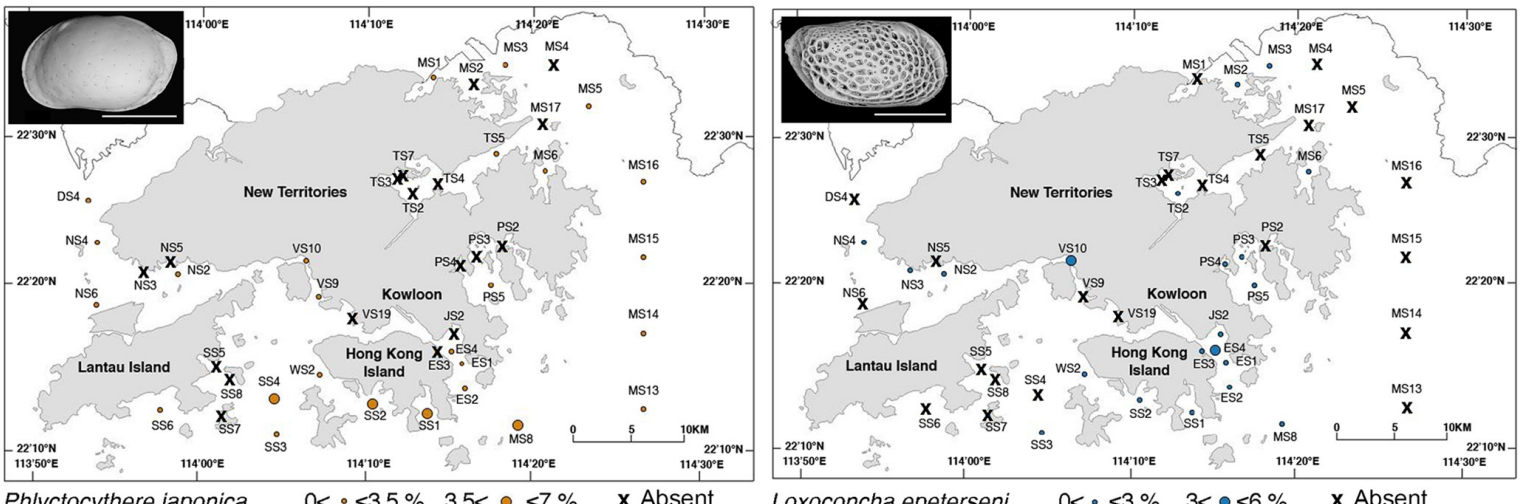

Phlyctocythere japonica $0<0 \leq 3.5 \% \quad 3.5<0 \leq 7 \% \quad \mathrm{x}$ Absent

Loxoconcha epeterseni $\quad 0<\bullet \leq 3 \% \quad 3<\bullet \leq 6 \% \quad$ x Absent
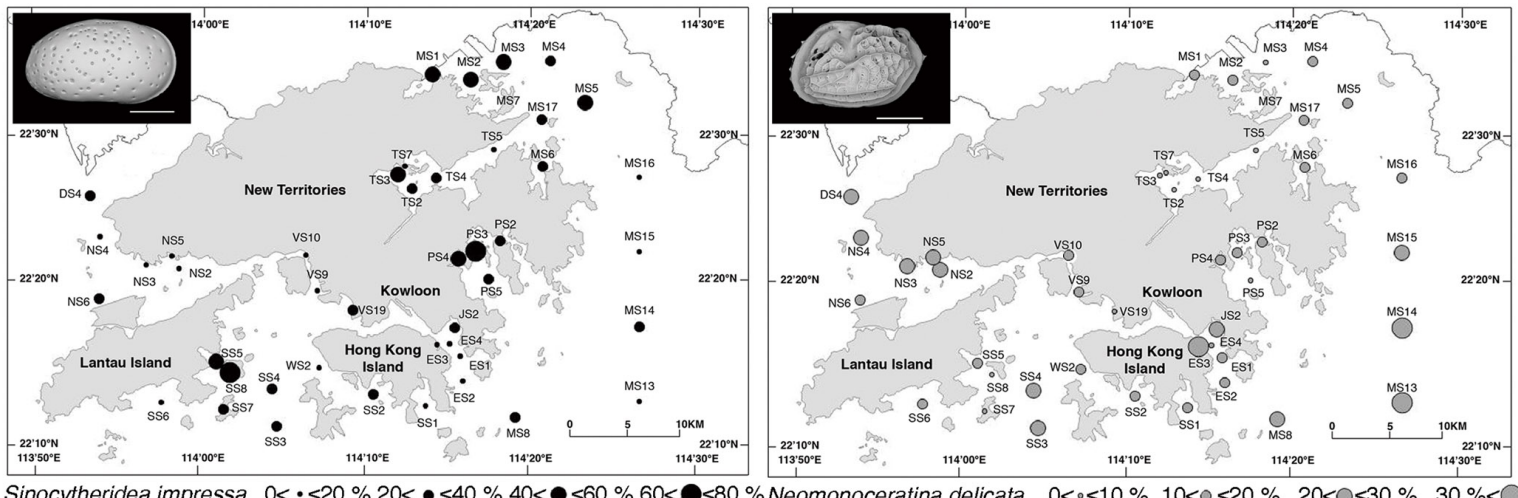

Sinocytheridea impressa $\quad 0<-\leq 20 \% 20<\bullet \leq 40 \% \quad 40<\bullet \leq 60 \% \quad 60<-80 \%$ Neomonoceratina delicata $\quad 0<\bullet \leq 10 \% \quad 10<0 \leq 20 \% \quad 20<0 \leq 30 \% \quad 30 \%<0$

Figure 5. Spatial distribution of the relative abundance for Pistocythereis bradyi, Bicornucythere bisanensis s.l., Nipponocythere bicarinata, Spinileberis quadriaculeata, Phlyctocythere japonica, Loxoconcha epeterseni, Sinocytheridea impressa, and Neomonoceratina delicata in Hong Kong. The scale bar is $200 \mu \mathrm{m}$. 
2012a). We did not see a significant relation between relative abundance and metal concentration, productivity, or dissolved oxygen. Possibly, the more dominant presence of $S$. impress $a$ and $N$. delicata, which are neither dominant or distributed throughout most of Japan, could explain this difference. These species may have a higher tolerance than B. bisanensis s.l. Another explanation may be that different morphotypes have different ecological preferences (Abe, 1988), and only Form A is known to be tolerant to eutrophication and bottom-water oxygen depletion (Irizuki et al., 2011, 2015a, 2018; Yasuhara and Yamazaki, 2005; Yasuhara et al., 2007). Form A is less abundant in Hong Kong, and due to the difficulty of juvenile identification, we did not divide $B$. bisanensis into morphotypes. Bicornucythere bisanensis s.l. is widely distributed throughout marginal marine environments around Japan, Russia (Sea of Japan coast), and the East and South China Sea (Fig. 4).

The relative abundance of $N$. bicarinata correlated with productivity (negative) (Tables 2 and 3). This is a typical middle bay species in Japan (Irizuki et al., 2006), abundant on muddy substrates at water depths $>10 \mathrm{~m}$ (Yasuhara and Seto, 2006; Yasuhara et al., 2005). We found N. bicarinata to be sensitive to eutrophication, preferring lower productivity (Figs. 2 and 5). This species is known from marginal marine environments around Japan and the East and South China Sea (Fig. 4).

The relative abundance of $S$. quadriaculeata correlated with productivity (positive) and turbidity (negative) (Tables 2 and 3). This is a typical inner muddy bay species in Japan (Irizuki et al., 2006), which prefers silty substrates in brackish waters at salinities from 20 to 30 and water depths of 2-7 m (Ikeya and Shiozaki, 1993). This study shows a preference for waters with higher productivity but relatively low turbidity (Tables 2 and 3) so that the species is abundant in Tolo Harbour (higher productivity, lower turbidity) but not in Deep Bay (higher turbidity) (Figs. 2 and 5). Spinileberis quadriaculeata is not tolerant to seasonal anoxia or oxygen depletion $\left(0-1 \mathrm{mg} \mathrm{L}^{-1}\right)$ in Uranouchi Bay, Japan (Irizuki et al., 2008), but we do not find a significant correlation with dissolved oxygen content, probably due to the relatively high bottom-water oxygen content $\left(2.96-6.84 \mathrm{mg} \mathrm{L}^{-1}\right)$ in Hong Kong (Fig. 2; Supplement S2). Spinileberis quadriaculeata is widely distributed in marginal marine environments around Japan, Russia (Sea of Japan coast), and the East and South China Sea (Fig. 4).

The relative abundance of $P$. japonica correlated with water depth (positive) (Tables 2 and 3). This species is known from relatively deeper waters $(>40 \mathrm{~m})$ in the East China Sea (Ishizaki, 1981; Wang et al., 1988). At our sites, it has its greatest abundance at the deeper southern sites (Fig. 5). Phlyctocythere japonica is distributed around Japan (Yasuhara et al., 2002) and the East and South China Sea (Fig. 4).

Similarly to $P$. japonica, the relative abundance of $L$. epeterseni correlated with water depth (positive) and turbidity (negative) (Tables 2 and 3). It occurs in the southern and eastern, deeper, and less turbid regions of Hong Kong waters, but the trend is not very clear (Figs. 2 and 5). This species is also known from the deeper parts of Osaka Bay (Yasuhara and Irizuki, 2001) and marginal marine environments around Japan (Ishizaki, 1968), the East China Sea (Hou et al., 1982), and the South China Sea (Cao, 1998) (Fig. 4). This species is reported as Loxoconcha modesta in Hou and Gou (2007) and has also been misidentified as Loxoconcha viva and Loxoconcha sinensis (Hou and Gou, 2007). Ishizaki (1968) described Loxoconcha laeta and Loxoconcha modesta, but these are the females and males of the same species (Ikeya et al., 2003). Ishizaki (1981) gave the new species names Loxoconcha epeterseni and Loxoconcha tosamodesta for Loxoconcha laeta and Loxoconcha modesta, respectively, because these names were junior homonyms. Since Loxoconcha laeta (epeterseni) appears earlier than Loxoconcha modesta (tosamodesta) in the original description (Ishizaki, 1968), we use the name Loxoconcha epeterseni for this species (see, e.g., Ikeya et al., 2003).

\subsection{Subtropical group}

Six species including Sinocytheridea impressa, Neomonoceratina delicata, Keijella kloempritensis, Neosinocythere elongata, Stigmatocythere roesmani, and Hemicytheridea reticulata are reported from the East China Sea to the IndoPacific area.

Sinocytheridea impressa (Sinocytheridea latiovata; see Whatley and Zhao, 1988a) is the most dominant species in this study, the relative abundance of which is significantly correlated with salinity (negative), dissolved oxygen (negative), mud content (positive), and productivity (positive) (Tables 2 and 3). This species is noticeably dominant in areas characterized by a muddy bottom including northern Mirs Bay, Port Shelter, and coastal southern waters (Fig. 5). It is also abundant in Tolo Harbour, an area known for its summer hypoxia and eutrophication (Hu et al., 2001; Sin and Chau, 1992). These results are consistent with previous studies indicating that $S$. impressa is dominant in low-salinity, nutrientrich, and turbid estuaries (Irizuki et al., 2005; Tanaka et al., 2011), but we did not see a significant relation with turbidity (Tables 2 and 3). Sinocytheridea impressa is known as a euryhaline species widely distributed throughout the East and South China Sea (abundant in water depths of $<20 \mathrm{~m}$; Whatley and Zhao, 1988a) and the Indo-Pacific (Fig. 4).

Neomonoceratina delicata (Neomonoceratina crispata; see Hou and Gou, 2007) is very common in most of the samples, and the relative abundance significantly correlates with $\mathrm{Zn}$ (positive), salinity (positive), and turbidity (positive) (Tables 2 and 3). Neomonoceratina delicata is a nearshore species abundant at depths less than $30 \mathrm{~m}$ at relatively high salinities (> 30; Zhao and Wang, 1988b). It prefers the higher-salinity waters in Hong Kong (Figs. 2 and 5; Tables 2 and 3 ) and in view of its positive correlation with $\mathrm{Zn}$ and 

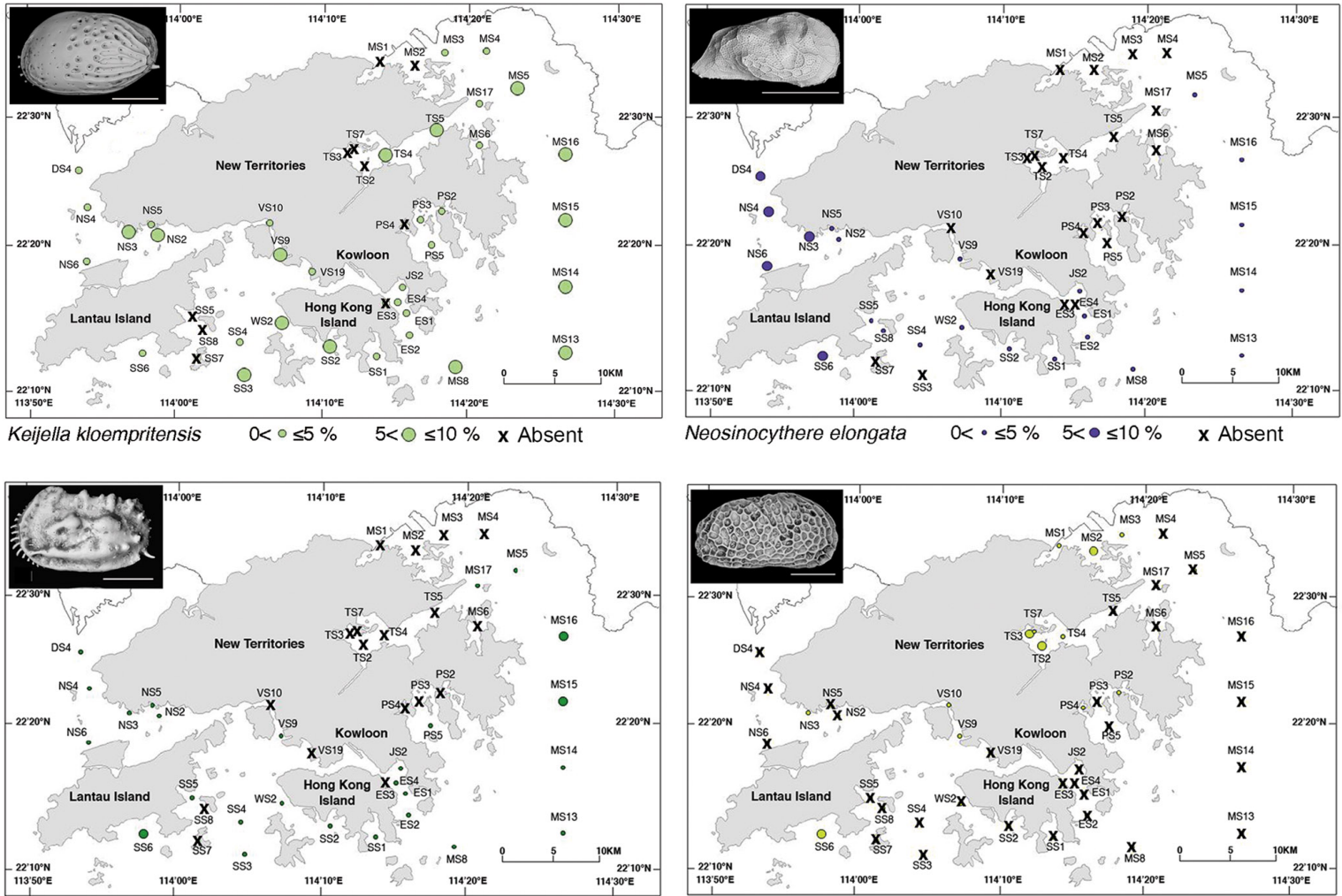

Neosinocythere elongata $\quad 0<\bullet \leq 5 \% \quad 5<\bullet \leq 10 \% \quad$ x Absent

Stigmatocythere roesmani $0<\cdot \leq 4 \% \quad 4<0 \leq 8 \% \quad$ x Absent
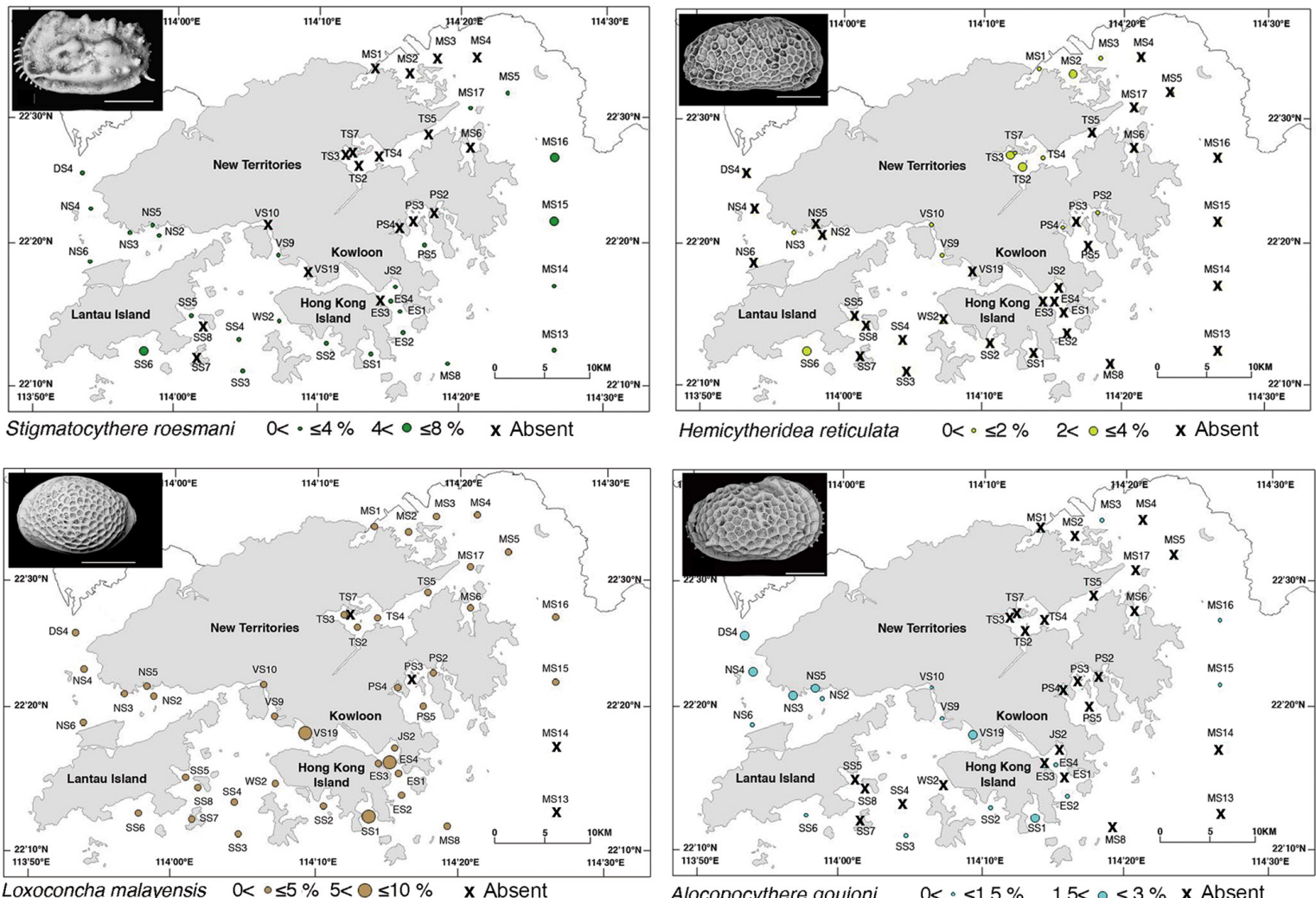

Hemicytheridea reticulata $\quad 0<0 \leq 2 \% \quad 2<0 \leq 4 \% \quad$ x Absent
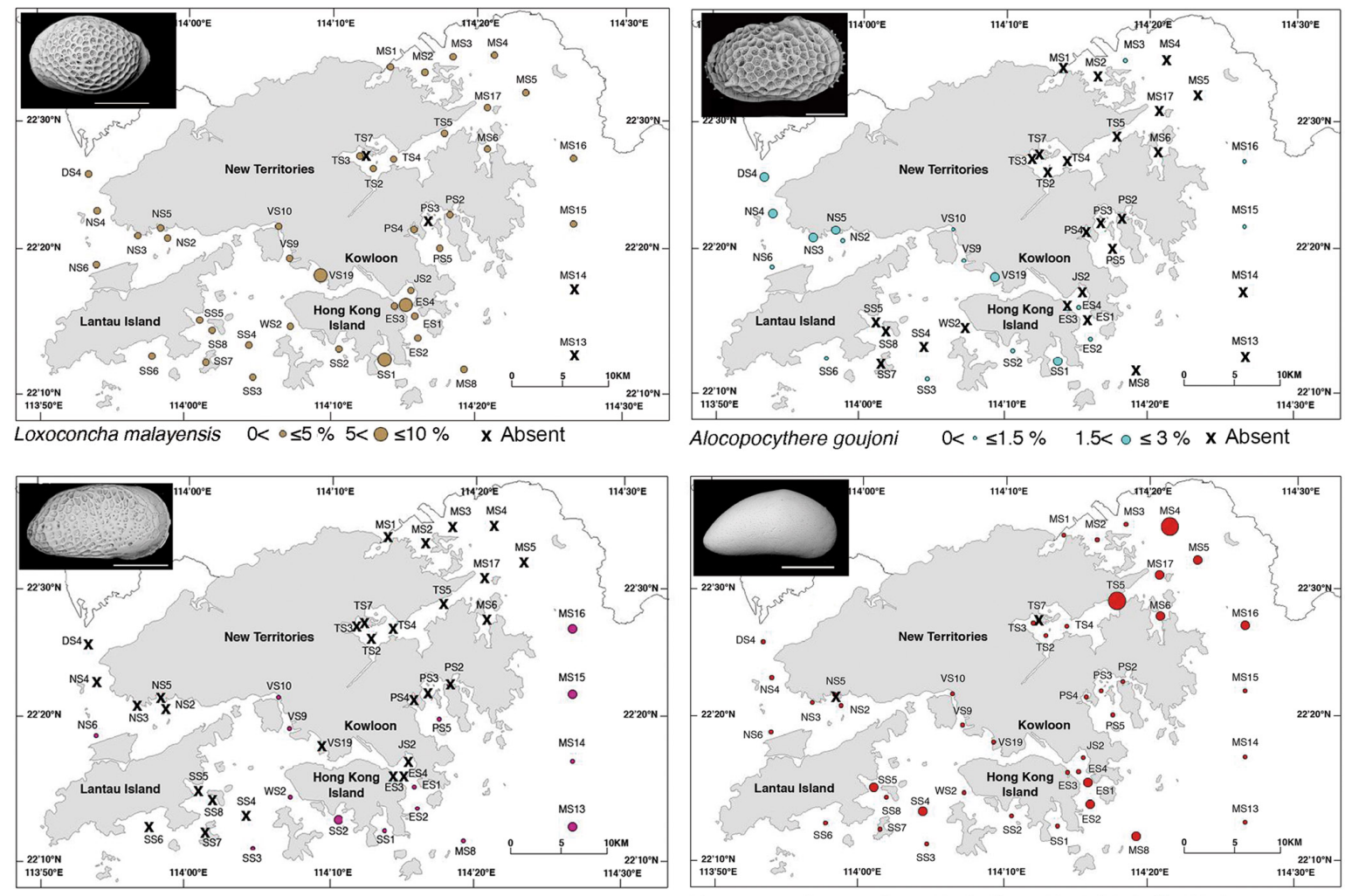

Hemikrithe orientalis $\quad 0<\bullet \leq 3 \% \quad 3<\bullet \leq 6 \%$ x Absent

Propontocypris spp. $\quad 0<\bullet \leq 10 \% \quad 10<0 \leq 20 \% 20 \%<0 \leq 60 \%$ x Absent

Figure 6. Spatial distribution of the relative abundance for Keijella kloempritensis, Neosinocythere elongata, Stigmatocythere roesmani, Hemicytheridea reticulata Loxoconcha malayensis, Alocopocythere goujoni, Hemikrithe orientalis, and Propontocypris spp. in Hong Kong. The scale bar is $200 \mu \mathrm{m}$. 
turbidity, is likely tolerant to human-induced environmental stress such as pollution and eutrophication. This species is widely known from nearshore and estuarine environments in the East and South China Sea and the Indo-Pacific (Fig. 4).

The relative abundance of $K$. kloempritensis correlated only with water depth (positive) (Fig. 6; Tables 2 and 3). Keijella kloempritensis is widely known from the tropical Indo-Pacific region and abundant along the inner continental shelf of the South and East China Sea (Fig. 4) at water depths ranging from 20 to $50 \mathrm{~m}$ and salinity close to normal marine (Zhao and Wang, 1990). Our modeling results are consistent with this, showing a preference for the relatively deeper waters in our study (Tables 2 and 3). Thus, this species is probably useful for reconstructing past sea-level changes in the broad tropical and subtropical Indo-Pacific and northwestern Pacific regions as a deeper water indicator.

The relative abundance of $N$. elongata correlated only with turbidity (positive) (Tables 2 and 3). This species occurs along the entire coast of China (Fig. 4) in marginal marine environments, especially estuarine environments shallower than $20 \mathrm{~m}$ (Dong et al., 2012; Hou and Gou, 2007; Liu et al., 2013, 2017; Zhao and Whatley, 1993) (Fig. 4), and is known from the Indo-Pacific region. Our modeling results and previous studies consistently indicate that $N$. elongata prefers shallow, turbid waters like Deep Bay and the Pearl River Estuary (Figs. 2 and 6).

The relative abundance of $S$. roesmani correlated with $\mathrm{Pb}$ (negative) (Tables 2 and 3), and thus this species is sensitive to metal pollution (but note the significant autocorrelation with the modeling result) and absent in areas with high metal concentrations, e.g., Tolo and Victoria Harbour (Figs. 3 and 6). The relative abundance of $H$. reticulata also correlated with $\mathrm{Pb}$ (negative) and water depth (negative) (Tables 2 and 3). This species is abundant in Tolo Harbour and the inner part of Mirs Bay (Fig. 6) at shallow depths and is also consistently found in very shallow waters from the Indo-Pacific (Zhao and Whatley, 1989). Their metal pollution sensitivity is contradictory because they occur in Tolo and Victora Harbour, both polluted regions of Hong Kong, and further research is needed to better understand this result (Figs. 3 and 6). Both of these species occur in the East and South China Sea and the Indo-Pacific region (Fig. 4).

\subsection{Tropical group}

The three species Loxoconcha malayensis, Alocopocythere goujoni, and Hemikrithe orientalis are distributed from the South China Sea to the Indo-Pacific.

The relative abundance of $L$. malayensis correlated with dissolved oxygen (negative) and mud content (negative) (Tables 2 and 3). It is a typical tropical species known from the Indo-Pacific and the South China Sea (Fig. 4). We did not find a correlation with temperature, likely due to the small range of variation of bottom-water temperatures in Hong Kong (winter temperature: $19.10-21.49^{\circ} \mathrm{C}$ ). This species prefers coarse sediments and is resistant to low oxygen content (Table 2 and 3), as seen by its abundance in Victoria Harbour (Figs. 2 and 6).

The relative abundance of both A. goujoni and $H$. orientalis correlated with natural factors only. Alocopocythere goujoni correlated with salinity (positive) (Tables 2 and 3) and occurs not only in Mirs Bay where the salinity is higher, but also in the Deep Bay and northwestern waters where the salinity is lower than in other areas (Figs. 2 and 6). The Deep Bay and northwestern waters are shallow and have relatively low oxygen content. The modeling result of this species shows a marginally insignificant but negative relationship to oxygen content and water depth with moderately high relative importance (Table 3). We explain this inconsistency by considering their preference for higher salinity and shallow water depths, and also their resistance to low oxygen conditions, but further research is needed to know their autoecology with better confidence. The relative abundance of H. orientalis correlated with water depth (positive) (Tables 2 and 3; but note the significant autocorrelation with the modeling result of this species), and it is more abundant in deeper waters including southern Mirs Bay (Fig. 6). It is known from depths of 20-50 $\mathrm{m}$ in the South China Sea (Zhao and Wang, 1988a) and reported from tropical Indo-Pacific marginal marine environments (Fig. 4). Our regression modeling consistently shows a positive relationship between relative abundance and winter temperatures with moderately high relative importance, although the correlation is marginally insignificant (Tables 2 and 3).

\subsection{Globally distributed group}

Propontocypris is known as a cosmopolitan genus. The relative abundance of Propontocypris spp. significantly correlated with productivity (negative) (Tables 2 and 3). This negative correlation with productivity (but note a significant autocorrelation with the modeling result of this genus) indicates that the genus prefers less eutrophic waters (Fig. 6). Propontocypris is a good swimmer (Maddocks, 1969) and thus may have an advantage in obtaining food in relatively food-limited environments.

Phytal genera including Neonesidea spp. and Xestoleberis spp. have a global distribution and are correlated with various environmental factors. The relative abundance of Neonesidea spp. correlated with dissolved oxygen (positive), as expected for a phytal species (Smith and Kamiya, 2002; Yamada, 2007) (Tables 2 and 3; Fig. 7). Similarly, phytal (Irizuki et al., 2008; Sato and Kamiya, 2007; Yasuhara et al., 2002) Xestoleberis spp. correlated with dissolved oxygen (positive), turbidity (negative), and mud content (negative) (Table 2 and 3; Fig. 7). This taxon's habitat preference, including clear water, coarse sediment, and high oxygen content, is reflected in our modeling. As mentioned above, the value of allochthonous phytal species to environmental interpretation 

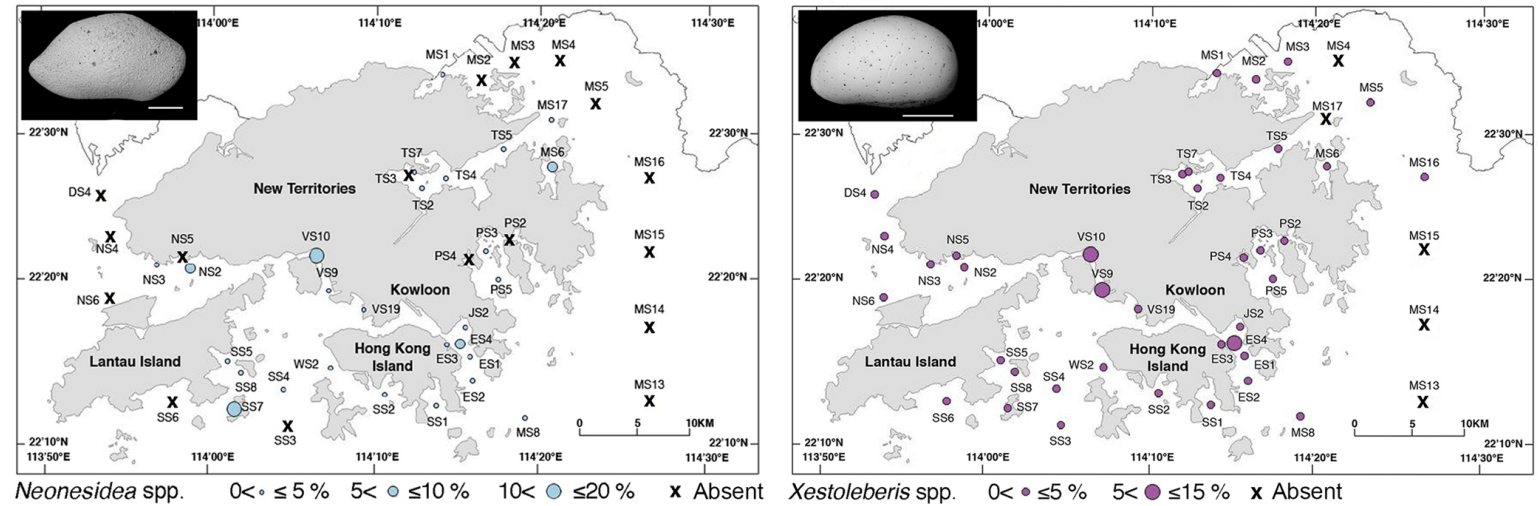

Figure 7. Spatial distribution of the relative abundance for Neonesidea spp. and Xestoleberis spp. in Hong Kong. The scale bar is $200 \mu \mathrm{m}$.

Table 5. Summary of autoecology for common ostracod taxa. Chl: chlorophyll $a$; D: water depth; DO: dissolved oxygen; MD: mud content; Sal: salinity; Tur: turbidity; WT: winter temperature; Pb: lead; Zn: zinc; R: region. The + and - marks indicate significant positive and negative correlations, respectively.

\begin{tabular}{|c|c|c|c|c|c|c|c|c|c|c|}
\hline Taxa & Chl & $\mathrm{D}$ & DO & MD & Sal & Tur & WT & $\mathrm{Pb}$ & $\mathrm{Zn}$ & $\mathrm{R}$ \\
\hline Pistocythereis bradyi & & - & & & + & & & - & & \\
\hline Bicornucythere bisanensis s.l. & & - & & & & & & & & \\
\hline Nipponocythere bicarinata & - & & & & & & & & & \\
\hline Spinileberis quadriaculeata & + & & & & & - & & & & \\
\hline Phlyctocythere japonica & & + & & & & & & & & \\
\hline Loxoconcha epeterseni & & + & & & & - & & & & \\
\hline Sinocytheridea impressa & + & & - & + & - & & & & & \\
\hline Neomonoceratina delicata & & & & & + & + & & & + & \\
\hline Keijella kloempritensis & & + & & & & & & & & \\
\hline Neosinocythere elongata & & & & & & + & & & & \\
\hline Stigmatocythere roesmani & & & & & & & & - & & \\
\hline Hemicytheridea reticulata & & - & & & & & & - & & \\
\hline Loxoconcha malayensis & & & - & - & & & & & & \\
\hline Alocopocythere goujoni & & & & & + & & & & & \\
\hline Hemikrithe orientalis & & + & & & & & & & & \\
\hline Propontocypris spp. & - & & & & & & & & & \\
\hline Neonesidea spp. & & & + & & & & & & & \\
\hline Xestoleberis spp. & & & + & - & & - & & & & \\
\hline
\end{tabular}

is limited, but they broadly reflect adjacent phytal environments.

\section{Summary}

Benthic ostracods from Hong Kong marginal marine waters studied here include widespread (i.e., one species distributed throughout the northwestern Pacific-Indo-Pacific region), temperate (i.e., five species distributed from the South China Sea to Japan and Russia), subtropical (i.e., six species distributed from the Indo-Pacific to the East China Sea), and tropical (i.e., three species distributed in the Indo-Pacific and South China Sea) species and three globally distributed genera (Fig. 4). We provide a robust baseline of the autoecology for these common ostracod taxa based on rigorous statistical modeling using comprehensive environmental data. We established reliable indicator taxa for water depth, mud content, salinity, turbidity, dissolved oxygen, heavy metal pollution $(\mathrm{Pb}$ and $\mathrm{Zn}$ ), and eutrophication (chlorophyll $a$ ) (Table 5). Thus, our results are applicable for future ostracod-based paleoenvironmental studies in a wide range of localities from the tropics to the extratropics and from the Indian Ocean to the northwestern Pacific. We established pollution and eutrophication indicator species in tropical environments for the first time. Anthropocene paleoenvironmental and paleoecological studies in the tropics are urgently needed because (1) the tropics are seriously under-studied (Wilkinson et al., 2014; Yasuhara et al., 2012a), (2) tropical environments and ecosystems are vulnerable and sensitive to human influences (Jackson et al., 2001; Pandolfi et al., 2003), and (3) Indo- 
Pacific tropical environments have been seriously degraded by human activity in rapidly developing countries (Bellwood et al., 2004; Jackson, 2008; Knowlton and Jackson, 2008). Our results provide useful and reliable tools for tropical Anthropocene research in the broad Indo-Pacific region.

Data availability. The data used are listed in the tables and the Supplement.

Supplement. The supplement related to this article is available online at: https://doi.org/10.5194/bg-16-585-2019-supplement.

Author contributions. MY and YH conceived and designed the research; YH, HI, MY, and BM performed the research; YH and MY analyzed the data; $\mathrm{YH}, \mathrm{MY}$, and BM wrote most of the paper.

Competing interests. The authors declare that they have no conflict of interest.

Acknowledgements. We thank the following: the Environmental Protection Department of Hong Kong, especially Kit Yung, for support; Laura Wong, Cecily Law, Maria Lo, and the staff of the Electronic Microscope Unit of the University of Hong Kong for their technical support; Bob Lin, Samuel Wang, Raymond Mak and Veta Wang for helping with sampling; Peter Frenzel, Mark Warne, Ellen Thomas and Oliver Friedrich for comments on an early version of the paper; S. Wajih A. Naqvi for editing; and Toshiaki Irizuki, Thomas M. Cronin, and Julio Rodríguez-Lázaro for reviewing our paper. The work described in this paper was partially supported by the Environment and Conservation Fund of Hong Kong (project code: 19/2012), the General Research Fund of the Research Grants Council of Hong Kong (project code: HKU 17303115), the Early Career Scheme of the Research Grants Council of Hong Kong (project code: HKU 709413P), and the Seed Funding Programme for Basic Research of the University of Hong Kong (project codes: 201111159140, 201611159053) (to Moriaki Yasuhara).

Edited by: S. Wajih A. Naqvi

Reviewed by: Toshiaki Irizuki, Thomas M. Cronin, and Julio Rodríguez-Lázaro

\section{References}

Abe, K.: Speciation completed in Keijella bisanensis species group, in: Evolutionary biology of Ostracoda-its fundamentals and applications, edited by: Hanai, T., Ikeya, N., and Ishizaki, K., Kodansha, Tokyo, Elsevier, Amsterdam, 11, 919-925, 1988.

Al Jumaily, W. A. K. and Al-Sheikhly, S. S.: Palaeozoogeography of shallow marine Ostracoda from Holocene sediments-southern Iraq, Qatar Univ. Sci. J., 18, 215-230, 1999.
Alberti, M., Hethke, M., Fürsich, F. T., and Liu, C.: Macroversus microfauna: resolution potential of bivalves, gastropods, foraminifera and ostracods in reconstructing the Holocene palaeoenvironmental evolution of the Pearl River delta, China, Palaeobio. Palaeoenv., 93, 327-353, 2013.

Anderson, D. R. and Burnham, K. P.: Avoiding pitfalls when using information-theoretic methods, J. Wildlife Manage., 66, 912918, 2002.

Anderson, D. R., Burnham, K. P., and Thompson, W. L.: Null hypothesis testing: problems, prevalence, and an alternative, J. Wildlife Manage., 64, 912-923, 2000.

Bartoń, K.: MuMIn: Multi-model inference, $\mathrm{R}$ package version 1.9.13, The Comprehensive R Archive Network (CRAN), available at: https://CRAN.R-project.org/package=MuMIn (last access: 3 September 2018), 2013.

Bellwood, D. R., Hughes, T. P., Folke, C., and Nyström, M.: Confronting the coral reef crisis, Nature, 429, 827-833, 2004.

Bivand, R. and Piras, G.: Comparing implementations of estimation methods for spatial econometrics, J. Stat. Softw., 63, 1-36, 2015.

Blackmore, G.: An overview of trace metal pollution in the coastal waters of Hong Kong, Sci. Total Environ., 214, 21-48, 1998.

Boomer, I. and Eisenhauer, G.: Ostracod faunas as palaeoenvironmental indicators in marginal marine environments, in: The Ostracoda: applications in Quaternary research, edited by: Holmes, J. A. and Chivas, A. R., vol. 131, American Geophysical Union, Washington DC, 135-149, 2002.

Burnham, K. P. and Anderson, D. R.: Model Selection and Multimodel Inference: A Practical Information-theoretic Approach, Springer, New York, 2002.

Cao, M.: Ostracods from Quaternary Hang Hau Formation, Lei Yue Mun, Hong Kong, in: Fossils and Strata of Hong Kong (Lower Volume), edited by: Li, Z., Chen, J., and He, G., Science Press, Beijing, 171-183, 1998 (in Chinese).

Cronin, T. M.: Ostracods and sea level, in: Handbook of Sea-Level Research, edited by: Shennan, I., Long, A. J. and Horton, B. P, John Wiley and Sons Ltd., 249-257, 2015.

Cronin, T. M. and Vann, C. D.: The sedimentary record of climatic and anthropogenic influence on the Patuxent estuary and Chesapeake Bay ecosystems, Estuaries, 26, 196-209, 2003.

Dewi, K. T.: Ostracoda from the Java Sea, west of Bawean Island, Indonesia, Mar. Geol. Inst. Spec. Pub., 4, 1-115, 1997.

Dong, Y., Liu, C., Chen, M., Qu, R., Wu, T., and Zhao, F.: Late Quaternary paleontology and environmental changes in Da'Ao plain middle Pearl River Delta, Quatern. Sci., 32, 1183-1198, 2012 (in Chinese with English Abstract).

Environmental Protection Department of Hong Kong: Marine Water Quality in Hong Kong 2011, available at: https://www.epd.gov. hk/epd/sites/default/files/epd/english/environmentinhk/water/ hkwqrc/files/waterquality/annual-report/marinereport2011.pdf (last access: 22 January 2019), 2011.

Fauzielly, L., Irizuki, T., and Sampei, Y.: Vertial changes of recent ostracode assemblages and environment in the inner part of Jakarta Bay, Indonesia, J. Coast. Develop., 16, 11-24, 2013.

Frenzel, P. and Boomer, I.: The use of ostracods from marginal marine, brackish waters as bioindicators of modern and Quaternary environmental change, Palaeogeogr. Palaeoclimatol. Palaecol., 225, 68-92, 2005. 
Gu, Y., Liu, H., and Qin, Y.: Postglacial transgression maximum documented by the core sediments of Xixi Wetland, East China, Quatern. Int., 436, 84-95, 2017.

Hazel, J. E.: Determining late Neogene and Quaternary palaeoclimates and palaeotemperature regimes using ostracods, in: Ostracoda in the Earth Sciences, edited by: De Deckker, P., Colin, J. P., and Peypouquet, J. P., Elsevier, Amsterdam, 89-103, 1988.

Hendrickx, J., Pelzer, B., Te Grotenhuis, M., and Lammers, J.: Collinearity involving ordered and unordered categorical variable, RC33 conference, Amsterdam, the Netherlands, 17-20 August, 2004.

Hodgkiss, I. J. and Yim, W. W. S.: A case study of Tolo Harbour, Hong Kong, in: Eutrophic Shallow Estuaries and Lagoons, edited by: McComb, A. J., CRC, Press, Baton Rouge, USA, 41-57, 1995.

Hoffman, J. I. E.: Biostatistics for Medical and Biomedical Practitioners, Academic Press, Tiburon, California, USA, 2015.

Hong, Y., Yasuhara, M., Iwatani, H., Seto, K., Yokoyama, Y., Yoshioka, K., and Mamo, B.: Freshwater reservoir construction by damming a marine inlet in Hong Kong: Paleoecological evidence of local community change, Mar. Micropaleontol., 132, 53-59, 2017.

Horne, D., Holmes, J., Viehberg, F., and Rodriguez-Lazaro, J.: Ostracoda as proxies for Quaternary climate change, in: Developments in Quaternary Science, edited by: Jaap, J. M. and van der Meer, J. J. M., vol. 17, Elsevier, Amsterdam, 1-337, 2012.

Hou, Y. T. and Gou, Y. X.: Ostracod Fossils of China, Vol. II, Cytheracea and Cytherellidae, Science Press, Beijing, 2007 (in Chinese).

Hou, Y., Chen, T., Yang, H., Ho, J., Zhou, Q., and Tian, M.: Cretaceous-Quaternary Ostracoda Fauna from Jiangsu, Geological Publishing House, Peking, 1-386, 1982.

Hu, J., Zhang, G., Li, K., Peng, P., and Chivas, A. R.: Increased eutrophication offshore Hong Kong, China during the past 75 years: evidence from high-resolution sedimentary records, Mar. Chem., 110, 7-17, 2008.

Hu, W. F., Lo, W., Chua, H., Sin, S. N., and Yu, P. H. F.: Nutrient release and sediment oxygen demand in a eutrophic land-locked embayment in Hong Kong, Environ. Int., 26, 369-375, 2001.

Hussain, S. M. and Mohan, S. P.: Recent Ostracoda from Adyar River Estuary, Chennai, Tamil Nadu, J. Palaeontol. Soc. Ind., 45, 25-32, 2000.

Hussain, S. M. and Mohan, S. P.: Distribution of recent benthic Ostracoda in Adyar river estuary, east coast of India, Indian J. Mar. Sci., 30, 53-56, 2001.

Hussain, S. M., Ravi, G., Mohan, S. P., and Rao, N. R.: Recent benthic Ostracoda from the inner shelf off Chennai, south east coast of India-implication of microenvironments, Env. Micropal. Microbiol. Microbenth., 1, 105-121, 2004.

Hussain, S. M., Mohan, S. P., and Jonathan, M. P.: Ostracoda as an aid in identifying 2004 tsunami sediments: a report from SE coast of India, Nat. Hazards., 55, 513-522, 2010.

Ikeya, N. and Shiozaki, M.: Characteristics of the inner bay ostracodes around the Japanese islands: The use of ostracodes to reconstruct paleoenvironments, Mem. Geol. Soc. Japan, 39, 15-32, 1993 (in Japanese with English abstract).

Ikeya, N., Tanaka, G., Tsukagoshi, A.: Ostracoda, in: The Database of Japanese Fossil Type Specimens Described During The 20th Century (Part 3), edited by: Ikeya, N., Hirano, H., and Oga- sawara, K., Palaeontological Society of Japan:, Tokyo, 37-131, 2003.

Irizuki, T., Nakamura, Y., Takayasu, K., and Sakai, S.: Faunal changes of Ostracoda (Crustacea) in Lake Nakaumi, southwestern Japan, during the last 40 years, Geosci. Rep. Shimane Univ., 22, 149-160, 2003 (in Japanese with English abstract).

Irizuki, T., Matsubara, T., and Matsumoto, H.: Middle Pleistocene Ostracoda from the Takatsukayama Member of the Meimi Formation, Hyogo Prefecture, western Japan - significance of the occurrence of Sinocytheridea impressa, Paleontol. Res., 9, 3754, 2005.

Irizuki, T., Takata, H., and Ishida, K.: Recent Ostracoda from Urauchi Bay, Kamikoshiki-jima Island, Kagoshima Prefecture, southwestern Japan, Laguna, 13, 13-28, 2006.

Irizuki, T., Seto, K., and Nomura, R.: The impact of fish farming and bank construction on Ostracoda in Uranouchi Bay on the Pacific coast of southwest Japan-Faunal changes between 1954 and 2002/2005, Paleonto. Res., 12, 283-302, 2008.

Irizuki, T., Taru, H., Taguchi, K., and Matsushima, Y.: Paleobiogeographical implications of inner bay Ostracoda during the Late Pleistocene Shimosueyoshi transgression, central Japan, with significance of its migration and disappearance in eastern Asia, Palaeogeogr. Palaeoclimatol. Palaecol., 271, 316-328, 2009.

Irizuki, T., Takimoto, A., Sako, M., Nomura, R., Kakuno, K., Wanishi, A., and Kawano, S.: The influences of various anthropogenic sources of deterioration on meiobenthos (Ostracoda) over the last 100 years in Suo-Nada in the Seto Inland Sea, southwest Japan, Mar. Pollut. Bull., 62, 2030-2041, 2011.

Irizuki, T., Ito, H., Sako, M., Yoshioka, K., Kawano, S., Nomura, R., and Tanaka, Y.: Anthropogenic impacts on meiobenthic Ostracoda (Crustacea) in the moderately polluted Kasado Bay, Seto Inland Sea, Japan, over the past 70 years, Mar. Pollut. Bull., 91, 149-159, 2015a.

Irizuki, T., Kobe, M., Ohkushi, K., Kawahata, H., and Kimoto, K.: Centennial-to millennial-scale change of Holocene shallow marine environments recorded in ostracode fauna, northeast Japan, Quatern. Res., 84, 467-480, 2015b.

Irizuki, T., Hirose, K., Ueda, Y., Fujihara, Y., Ishiga, H., and Seto, K.: Ecological shifts due to anthropogenic activities in the coastal seas of the Seto Inland Sea, Japan, since the 20th century, Mar. Pollut. Bull., 127, 637-653, 2018.

Ishizaki, K.: Ostracodes from Uranouchi Bay, Kochi Prefecture, Japan, Sci. Rep. Tohoku Univ. (2nd Ser Geol.), 40, 1-45, 1968.

Ishizaki, K.: Ostracoda from the East China Sea, Sci. Rep. Tohoku Univ. (2nd Ser Geol.), 51, 37-A39, 1981.

Iwasaki Y.: Ostracod assemblages from the Holocene deposits of Kumamoto, Kyushu, Kumamoto Jour. Sci. (Geol.), 12, 1-12, 1992 (in Japanese with English abstract).

Iwatani, H., Young, S. M., Irizuki, T., Sampei, Y., and Ishiga, H.: Spatial variations in recent ostracode assemblages and bottom environments in Trincomalee Bay, northeast coast of Sri Lanka, Micropaleontology, 509-518, 2014.

Jackson, J. B. C.: Ecological extinction and evolution in the brave new ocean, P. Natl. Acad. Sci. USA, 106, 19685-19692, 2008.

Jackson, J. B. C., Kirby, M. X., Berger, W. H., Bjorndal, K. A., Botsford, L. W., Bourque, B. J., Bradbury, R. H., Cooke, R., Erlandson, J., and Estes, J. A.: Historical overfishing and the recent collapse of coastal ecosystems, Science, 293, 629-637, 2001. 
Knowlton, N. and Jackson, J. B. C.: Shifting baselines, local impacts, and global change on coral reefs, PLoS Biol., 6, e54, https://doi.org/10.1371/journal.pbio.0060054, 2008.

Legendre, P. and Legendre, L.: Numerical Ecology: second English edition, vol. 20, Elsevier, Amsterdam, the Netherlands, 1998.

Li, S.: Distribution of the ostracod thanatocoenses in the Pearl River Mouth area, J. Tropic. Oceanogra., 4, 43-54, 1985.

Liu, C., Franz T., F., Wu, J., Dong, Y., Yang, T., Yin, J., Wang, Y., and Liu, M.: Late Quaternary palaeoenvironmental changes documented by microfaunas and shell stable isotopes in the southern Pearl River Delta plain, South China, J. Palaeogeogra., 2, 344 361, 2013

Liu, J., Liu, C., Jia, L., Yang T., Zhang, S., and Yin, J.: Foraminiferal and ostracod distribution in surface sediments from Huangmao Bay of Pearl River Estuary and its influencing factors, J. Palaeogeogr, 15, 413-422, 2013 (in Chinese with English abstract).

Liu, J., Zhang, X., Mei, X., Zhao, Q., Guo, X., Zhao, W., Liu, J., Saito, Y., Wu, Z., and Li, J.: The sedimentary succession of the last $\sim 3.50 \mathrm{Myr}$ in the western south Yellow Sea: Paleoenvironmental and tectonic implications, Mar. Geol., 399, 47-65, 2017.

Maddocks, R. F.: Recent ostracodes of the Family Pontocyprididae chiefly from the Indian Ocean, Smithson. Contrib. Zool., 7, 1-56, 1969.

Morton, B.: The subsidiary impacts of dredging (and trawling) on a subtidal benthic molluscan community in the southern waters of Hong Kong, Mar. Pollut. Bull., 32, 701-710, 1996.

Morton, B. and Wu, S.: The hydrology of the coastal waters of Hong Kong, Environ. Res., 10, 319-347, 1975.

Morton, B. and Blackmore, G.: South China Sea, Mar. Pollut. Bull., 42, 1236-1263, 2001.

Mostafawi, N.: Recent ostracods from the central Sunda Shelf, between the Malay Peninsula and Borneo, Senck. Leth., 72, 129168, 1992.

Nishath, N. M., Hussain, S. M., Neelavnnan, K., Thejasino, S., Saalim, S., and Rajkumar, A.: Ostracod biodiversity from shelf to slope oceanic conditions, off central Bay of Bengal, India, Palaeogeogr. Palaeoclimatol. Palaecol., 483, 70-82, 2017.

Noraswana, N. F., Ramlan, O., and Norashikin, S.: Distribution of recent Ostracoda in offshore sediment of selected stations in the Sulu Sea, Sabah, Malaysian App. Biol., 43, 49-57, 2014.

Owen, R. B. and Sandhu, N.: Heavy metal accumulation and anthropogenic impacts on Tolo Harbour, Hong Kong, Mar. Pollut. Bull., 40, 174-180, 2000.

Ozawa, H., Kamiya, T., Itoh, H., and Tsukawaki, S.: Water temperature, salinity ranges and ecological significance of the three families of Recent cold-water ostracods in and around the Japan Sea, Paleontol. Res., 8, 11-28, 2004.

Pandolfi, J. M., Bradbury, R. H., Sala, E., Hughes, T. P., Bjorndal, K. A., Cooke, R. G., McArdle, D., McClenachan, L., Newman, M. J. H., and Paredes, G.: Global trajectories of the long-term decline of coral reef ecosystems, Science, 301, 955-958, 2003.

Pugliese, N., Maria Eugenia, M., Francesco, S., and Chaimanee, N.: Environmental monitoring through the shallow marine ostracods of Phetchaburi area (NW Gulf of Thailand), in: Proceedings of the Second and Third Italian Meetings on Environmental Micropaleontology, Urbino, Italy, 10-12 June 2003 and 8-9 June 2004, 11, 85-90, 2006.
R Core Team.: R: A language and environment for statistical computing, Computer software 3.2.4, available at http://www. R-project.org/ (last access: 3 September 2018), 2016.

Ruiz, F., Abad, M., Bodergat, A. M., Carbonel, P., RodríguezLázaro, J., and Yasuhara, M.: Marine and brackish-water ostracods as sentinels of anthropogenic impacts, Earth-Sci. Rev., 72, 89-111, 2005.

Sato, T. and Kamiya, T.: Taxonomy and geographical distribution of recent Xestoleberis species (Cytheroidea, Ostracoda, Crustacea) from Japan, Paleontol. Res., 11, 183-227, 2007.

Schornikov, E. I., Zenina, M. A., and Ivanova, E. V.: Ostracods as indicators of the aquatic environmental conditions on the northeastern Black Sea shelf over the past 70 years, Russ. J. Mar. Biol., 40, 455-464, 2014.

Shin, P. K. S.: Changes in benthic infaunal communities in Tolo Harbour: will the trend continue, in: Proceedings of an International Workshop Reunion Conference, Hong Kong, 21-26 October 2001, 579-592, 2001.

Sin, Y. S. and Chau, K. W.: Eutrophication studies on Tolo Harbour, Hong Kong, Water Sci. Technol., 26, 2551-2554, 1992.

Smith, R. J. and Kamiya, T.: The ontogeny of Neonesidea oligodentata (Bairdioidea, Ostracoda, Crustacea), Hydrobiologia, 489, 245-275, 2002.

Stepanova, A., Taldenkova, E., and Bauch, H. A.: Recent Ostracoda from the Laptev Sea (Arctic Siberia): species assemblages and some environmental relationships, Mar. Micropaleontol., 48, 23 48, 2003.

Tanaka, G., Komatsu, T., and Phong, N. D.: Recent ostracod assemblages from the northeastern coast of Vietnam and the biogeographical significance of the euryhaline species, Micropaleontology, 55, 365-382, 2009.

Tanaka, G., Komatsu, T., Saito, Y., Nguyen, D. P., and Vu, Q. L.: Temporal changes in ostracod assemblages during the past 10,000 years associated with the evolution of the Red River delta system, northeastern Vietnam, Mar. Micropaleontol., 81, 77-87, 2011.

Tanaka, G., Henmi, Y., Masuda, T., Moriwaki, H., Komatsu, T., Zhou, B., Maekawa, T., Niiyama, S., Nguyen, P. D., and Doan, H. D.: Recent ostracod distribution in western Kyushu, Japan, related to the migration of Chinese continental faunal elements, Mar. Micropaleontol., 146, 1-38, 2019.

Tanner, P. A., Leong, L. S., and Pan, S. M.: Contamination of heavy metals in marine sediment cores from Victoria Harbour, Hong Kong, Mar. Pollut. Bull., 40, 769-779, 2000.

Wang, H. E., Zhang, H., Cao, M., and Horne, D. J.: Holocene Ostracods from the Hang Hau Formation in Lei Yue Mun, Hong Kong, and their palaeoenvironmental implications, Alcheringa, 1-11, https://doi.org/10.1080/03115518.2018.1511830, 2018.

Wang, P. and Zhao, Q.: Ostracod distribution in bottom sediments of the East China Sea, in: Marine Micropaleontology of China, edited by: Wang, P., China Ocean Press, Beijing, 70-92, 1985 (in Chinese).

Wang, P., Zhang, J., Zhao, Q., Min, Q., Bian, Y., Zheng, L., Cheng, X., and Chen, R.: Foraminifera and Ostracoda in Bottom Sediments of the East China Sea, Ocean Press, Beijing, 1988 (in Chinese with English abstract).

Wang, Q. and Zhang, L.: Holocene ostracod fauna and paleoenvironment in the sea region around Hong Kong, Acta Oceanol. Sin., 6, 281-291, 1987. 
Whatley, R. and Zhao, Q.: A revision of Brady's 1869 study of the Ostracoda of Hong Kong, J. Micropalaeontol., 7, 21-29, 1988a.

Whatley, R. and Zhao, Q.: Recent Ostracoda of the Malacca Straits, Part II, Rev. Española Micropaleontol. 20, 5-37, 1988b.

Wilkinson, I. P., Poirier, C., Head, M. J., Sayer, C. D., and Tibby, J.: Microbiotic signatures of the Anthropocene in marginal marine and freshwater palaeoenvironments, Geological Society, London, Special Publications, 395, 185-219, 2014.

Woodroffe, C. D., Nicholls, R. J., Saito, Y., Chen, Z., and Goodbred, S. L.: Landscape variability and the response of Asian megadeltas to environmental change, in: Global Change and Integrated Coastal Management: the Asia-Pacific Region, edited by: Harvey, N., Springer, Berlin, 10, 277-314, 2006.

Yamada, S.: Ultrastructure of the carapace margin in the Ostracoda (Arthropoda: Crustacea), Hydrobiologia, 585, 201-211, 2007.

Yasuhara, M. and Irizuki, T.: Recent Ostracoda from the northeastern part of Osaka Bay, southwestern Japan, J. Geosci. Osaka City Univ., 44, 57-95, 2001.

Yasuhara, M. and Seto, K.: Holocene relative sea-level change in Hiroshima Bay, Japan: A semi-quantitative reconstruction based on ostracodes, Paleontol. Res., 10, 99-116, 2006.

Yasuhara, M. and Yamazaki, H.: The impact of 150 years of anthropogenic pollution on the shallow marine ostracode fauna, Osaka Bay, Japan, Mar. Micropaleontol., 55(1), 63-74, 2005.

Yasuhara, M., Irizuki, T., Yoshikawa, S., and Nanayama, F.: Changes in Holocene ostracode faunas and depositional environments in the Kitan Strait, southwestern Japan, Paleontol. Res., 6, 85-99, 2002.

Yasuhara, M., Yamazaki, H., Irizuki, T., and Yoshikawa, S.: Temporal changes of ostracode assemblages and anthropogenic pollution during the last 100 years, in sediment cores from Hiroshima Bay, Japan, The Holocene, 13, 527-536, 2003.

Yasuhara, M., Yoshikawa, S., and Nanayama, F.: Reconstruction of the Holocene seismic history of a seabed fault using relative sealevel curves reconstructed by ostracode assemblages: Case study on the Median Tectonic Line in Iyo-nada Bay, western Japan, Palaeogeogr. Palaeoclimatol., 222, 285-312, 2005.

Yasuhara, M., Yamazaki, H., Tsujimoto, A., and Hirose, K.: The effect of long-term spatiotemporal variations in urbanizationinduced eutrophication on a benthic ecosystem, Osaka Bay, Japan, Limnol. Oceanogr., 52, 1633-1644, 2007.

Yasuhara, M., Hunt, G., Cronin, T. M., and Okahashi, H.: Temporal latitudinal-gradient dynamics and tropical instability of deep-sea species diversity, P. Natl. Acad. Sci. USA, 106, 21717-21720, 2009.
Yasuhara, M., Hunt, G., Breitburg, D., Tsujimoto, A., and Katsuki, K.: Human-induced marine ecological degradation: micropaleontological perspectives, Ecol. Evol., 2, 3242-3268, 2012a.

Yasuhara, M., Hunt, G., van Dijken, G., Arrigo, K. R., Cronin, T. M., and Wollenburg, J. E.: Patterns and controlling factors of species diversity in the Arctic Ocean, J. Biogeogr., 39, 20812088, 2012b.

Yasuhara, M., Iwatani, H., Hunt, G., Okahashi, H., Kase, T., Hayashi, H., Irizuki, T., Aguilar, Y. M., Fernando, A. G. S., and Renema, W.: Cenozoic dynamics of shallow-marine biodiversity in the Western Pacific, J. Biogeogr, 44, 567-578, 2017.

Zhao, Q.: Recent Ostracoda from the coast zone of the East China Sea and the Yellow Sea, Mar. Geol. Quatern. Geol., 4, 45-57, 1984 (in Chinese).

Zhao, Q. and Wang, P.: Distribution of modern Ostracoda in the shelf seas off China, in: Developments in Palaeontology and Stratigraphy, edited by: Hanai, T., Ikeya, N., and Ishizaki, K., Kodansha, Tokyo, Elsevier, Amsterdam, 11, 805-821, 1988a (in Chinese with English abstract).

Zhao, Q. and Wang, P.: Modern Ostracoda in sediments of shelf seas off China: quantitative and qualitative distributions, Oceanol. Limnol. Sin., 19, 553-561, 1988b (in Chinese with English abstract).

Zhao, Q. and Wang, P.: Modern Ostracoda in shelf seas off China: Zoogeographical zonation, Oceanol. Limnol. Sin., 21, 458-464, 1990 (in Chinese with English abstract).

Zhao, Q. and Whatley, R.: Recent podocopid Ostracoda of the Sedili River and Jason Bay, southeastern Malay Peninsula, Micropaleontology, 35, 168-187, 1989.

Zhao, Q. and Whatley, R.: New species of the ostracod genus Neosinocythere Huang (1985) from the IndoWest Pacific Region, J. Micropalaeontol., 12, 1-7, https://doi.org/10.1144/jm.12.1.1, 1993.

Zhao, Q., Wang, P., and Zhang, Q.: Ostracoda distribution in northern South China Sea continental Shelf, Acta Oceanol. Sin., 8, 590-602, 1986 (in Chinese).

Zhou, Y., Xie, Y., Chen, F., Long, G., and Chen, C.: Microfaunas in hole Zk201-2 at Zhujiang River Delta since Late Pleistocene and their implications for paleoenvironments, Mar. Geol. Quatern. Geol., 35, 114-123, 2015 (in Chinese with English abstract). 